\title{
Radial and vertical epicyclic frequencies of Keplerian motion in the field of Kerr naked singularities
}

\section{Comparison with the black hole case and possible instability of naked-singularity accretion discs}

\author{
G. Török and Z. Stuchlík
}

\author{
Institute of Physics, Faculty of Philosophy and Science, Silesian University in Opava, Bezručovo nám. 13, 74601 Opava, \\ Czech Republic \\ e-mail: stu10uf@fpf.slu.cz; terek@volny.cz
}

Received 6 February 2005 / Accepted 8 March 2005

\begin{abstract}
Relativistic Keplerian orbital frequency $\left(v_{\mathrm{K}}\right)$ and related epicyclic frequencies (radial $v_{\mathrm{r}}$, vertical $\left.v_{\theta}\right)$ play an important role in the physics of accretion discs orbiting Kerr black holes - quasiperiodic oscillations observed in microquasars can be explained by associated resonant or trapping effects. Because of growing theoretical evidence of the possible existence of naked singularities, we discuss the behaviour of the fundamenal orbital frequencies for Keplerian motion in the field of Kerr naked singularities, primarily in order to find phenomena that could observationally distinguish a hypothetical naked singularity from black holes. Some astrophysically important consequences are sketched, namely the existence of strong resonant frequency for all Kerr naked singularities, with radial and vertical epicyclic frequencies equal and given by the relation $\omega_{\mathrm{sr}}=a^{-2} \sqrt{a^{2}-1}\left(a^{2}+1\right)^{-1}$.
\end{abstract}

Key words. black holes physics - X-rays: general

\section{Introduction}

Quasiperiodic oscillations ( $Q P O s$ ) of X-ray brightness have been observed at low- $(\mathrm{Hz})$ and high- $(\mathrm{kHz})$ frequencies in some low-mass X-ray binaries containing neutron stars or black holes; for a review articles see, e.g., McClintock \& Remillard (2003) in the case of black hole binaries and van der Klis (2000) in the case of neutron star binaries. Since the peaks of high frequencies are close to the orbital frequency of the marginally stable circular geodesic representing the inner edge of Keplerian discs orbiting black holes (or neutron stars), strong gravity effects must be relevant if trying to explain high frequency QPOs (Abramowicz et al. 2004). In the context of discs oscillations (Okazaki et al. 1987; Nowak \& Wagoner 1991, 1992) both the warped discs (trapped) oscillations (Kato \& Fukue 1980; Kato 2004a) and resonant oscillations (Abramowicz \& Kluźniak 2001; Abramowicz et al. 2004) has been considered for explaining QPOs.

Usually, in the $\mathrm{kHz}$ QPOs the power spectrum shows twin peaks with frequencies correlated to their X-ray intensity and the peak separation almost constant (see, e.g., Strohmayer et al. 1996; Ford et al. 1997; Zhang et al. 1988; van der Klis et al. 1997). In the case of microquasars containing stellar mass black holes, the observed ratio of the twin peak frequencies is exactly, or almost exactly, 3:2; therefore, some resonant effects are probably involved in oscillating accretion discs of microquasars (Kluźniak \& Abramowicz 2000; Kluzniak \& Abramowicz 2001) ${ }^{1}$. It was shown that the parametric resonance of vertical and radial oscillations at epicyclic frequencies related to the Keplerian motion could be the most probable explanation of the observed microquasars phenomena (Török et al. 2005). On the other hand, the forced resonance of the epicyclic frequencies or some other kind of resonance with ratios given by small integral numbers, e.g., 2:1, 3:1, 5:2, etc. could also explain observed QPOs frequencies (with the same $3: 2$ ratio), if combinational ("beat") frequencies are considered (Abramowicz \& Kluźniak 2001; Török et al. 2005; Aschenbach 2004). The puzzle of this $3: 2$ ratio $\mathrm{kHz}$ frequencies has still not been definitely solved and other possible explanations, like warped-disc oscillations (see Kato 2004b) or simple $p$-mode oscillations (Rezzolla 2004), can not be exluded.

At any rate, strong observational evidence supports the astrophysical relevance of the disc oscillation concept introduced during the eighties (for a review of the concept itself see, e.g., Kato et al. 1998). The mechanisms for triggering

\footnotetext{
${ }^{1}$ Interestingly, the same 3:2 ratio seems to be present in the case of neutron stars sources, indicating the same origin of the observed quasiperiodic oscillations (Abramowicz et al. 2003; see however, Belloni et al. 2004).
} 
the oscillations in epicyclic frequencies were treated successfully both for thin (see Kato et al. 1998) and thick discs (e.g., Matsumoto et al. 1989; Abramowicz et al. 2003; Rezzolla 2004). Nevertheless, sophisticated three-dimensional magnetohydrodynamic simulations (3-MHD) of accretion flows usually do not show any twin peak $\mathrm{kHz}$ QPOs resembling those observed (Igumenshchev et al. 2003; De Villiers et al. 2003, and others). Only very recently Kato (2004c) has reported a view of the 3:2 twin peaks in 3-MHD simulations. In addition, it has recently been shown by Bursa et al. (2004) that the possible resonant oscillations of the torus could be directly observable in X-ray modulation when they occur in the inner parts of accretion flow around a black hole or neutron star, even if the source of radiation is steady and perfectly axisymmetric.

Apparently, the vertical and radial epicyclic frequencies of the Keplerian motion play a crucial role for both thin Keplerian discs and thick toroidal discs. Their properties have been extensively studied in the case of accretion discs orbiting Kerr black holes both in works mentioned above and in many others, yet continue to be very hot outstanding topic in recent astrophysics. On the other hand, it is natural to extend the concept of disc oscillations in the epicyclic frequencies around other physical objects.

According to the cosmic censorship hypothesis (Penrose 1969) and the uniqueness black-hole theorems (Carter 1973), the result of the gravitational collapse of a sufficiently massive rotating body is a rotating Kerr black hole, rather than a Kerr naked singularity. Although the cosmic censorship is a plausible hypothesis, there is some evidence against it. Naked singularities arise in various models of spherically symmetric collapse (e.g., Lake \& Zannias 1990; Joshi et al. 2002, 2004). In modelling the collapse of rotating stars, it was shown that in some situations mass shedding and gravitational radiation will not reduce the angular momentum of the star enough to lead to the formation of a Kerr black hole (Miller \& de Felice 1985). Candidates for the formation of Kerr naked singularities with a ring singularity were found in the scenario of Charlton \& Clarke (1990), while some 2D numerical models of collapsing, rotating supermassive objects imply that a Kerr-like naked singularity could develop from objects when rotating rapidly enough (Nakamura et al. 1987).

It is generally believed that black holes are stable against perturbations that would transfer them into naked singularities (Bardeen 1973; Thorne 1974; Wald 1974; Cohen \& Gautreau 1979; Israel 1986; de Felice \& Yu 1986). However, recently presented gedanken experiments concerning electrically charged, Reissner-Nordström black holes put this belief in doubt. It was shown that a charged test particle radially falling into a nearly Reissner-Nordström black hole could transform it into a Reissner-Nordström naked singularity (Hubeny 1999; see however, Quinn \& Wald 1999 for a more detailed analysis). Furthermore, it was shown that an extreme Reissner-Nordström black hole could be transformed into a Kerr-Newman naked singularity by capturing a flat and electrically neutral spinning body that plunges in radially with its spin aligned to the radial direction (de Felice \& Yu 2001). Moreover, the possible existence of naked singularities is supported by general mathematical studies concerning scalar fields around ReissnerNordström naked singularities (see, e.g., Stalker \& Shadi 2004).

Because the cosmic censorship hypothesis is far from being proved, naked-singularity spacetimes related to the blackhole spacetimes with a nonzero charge and/or rotation parameter could be considered conceivable models for some exotic Galactic binary systems or, on much higher scale, of quasars and active galactic nuclei, so they, too, deserve some attention. Of particular interest are those effects that could observationally distinguish a naked singularity from black holes. Therefore, we shall discuss here in detail the properties of the vertical and radial epicyclic frequencies of the Keplerian circular motion in the field of Kerr naked singularities, in order to find astrophysically relevant differencies between the blackhole and naked-singularity cases. Indeed, we will show that in the Kerr naked singularity spacetimes a new and astrophysically very important effects exist, which does not occur in the case of black holes; namely, for any Kerr naked singularity a stable circular geodesic exists on which the vertical and radial epicyclic frequencies are equal thus indicating the possibility of extremely strong resonance and instability of the Keplerian accretion discs.

In Sect. 2 we present the well-known formulae giving the vertical and radial epicyclic frequencies of the Keplerian circular orbits in the Kerr spacetimes. In Sect. 3 we discuss the properties of these frequencies for both black-hole and nakedsingularity spacetimes. We focus attention on the extrema of both the radial and vertical frequencies, if they exist. In Sect. 4, the naked-singularity case is discussed in detail, and the strong resonant frequency, when the epicyclic frequencies are equal, is given as a function of the naked singularity rotational parameter. In Sect. 5 we present some concluding remarks.

\section{Epicyclic oscillations of Keplerian discs}

In the case of oscillating Keplerian discs three orbital frequencies are relevant: Keplerian orbital frequency $\nu_{\mathrm{K}}=\Omega_{\mathrm{K}} / 2 \pi$, radial epicyclic frequency $v_{\mathrm{r}}=\omega_{\mathrm{r}} / 2 \pi$, and vertical epicyclic frequency $v_{\theta}=\omega_{\theta} / 2 \pi$. For discs orbiting Kerr black holes or naked singularities, corresponding angular velocities $\Omega_{\mathrm{K}}, \omega_{\mathrm{r}}, \omega_{\theta}$ are given by the well-known formulae (e.g., Nowak \& Lehr 1999),

$$
\begin{aligned}
\Omega_{\mathrm{K}} & =\left(\frac{G M_{0}}{r_{G}^{3}}\right)^{1 / 2}\left(x^{3 / 2}+a\right)^{-1}, \\
\omega_{\mathrm{r}}^{2} & =\alpha_{\mathrm{r}} \Omega_{\mathrm{K}}^{2} \\
\omega_{\theta}^{2} & =\alpha_{\theta} \Omega_{\mathrm{K}}^{2}
\end{aligned}
$$

where

$\alpha_{\mathrm{r}}(x, a) \equiv 1-6 x^{-1}+8 a x^{-3 / 2}-3 a^{2} x^{-2}$,

$\alpha_{\theta}(x, a) \equiv 1-4 a x^{-3 / 2}+3 a^{2} x^{-2}$,

and $x$ is introduced as dimensionless radial coordinate

$x=r / M$.

We use Boyer-Lindquist coordinates, $(t, r, \theta, \phi)$. We rescale the central object mass with $M=G M_{0} / c^{2}=r_{\mathrm{G}}$ and the central 

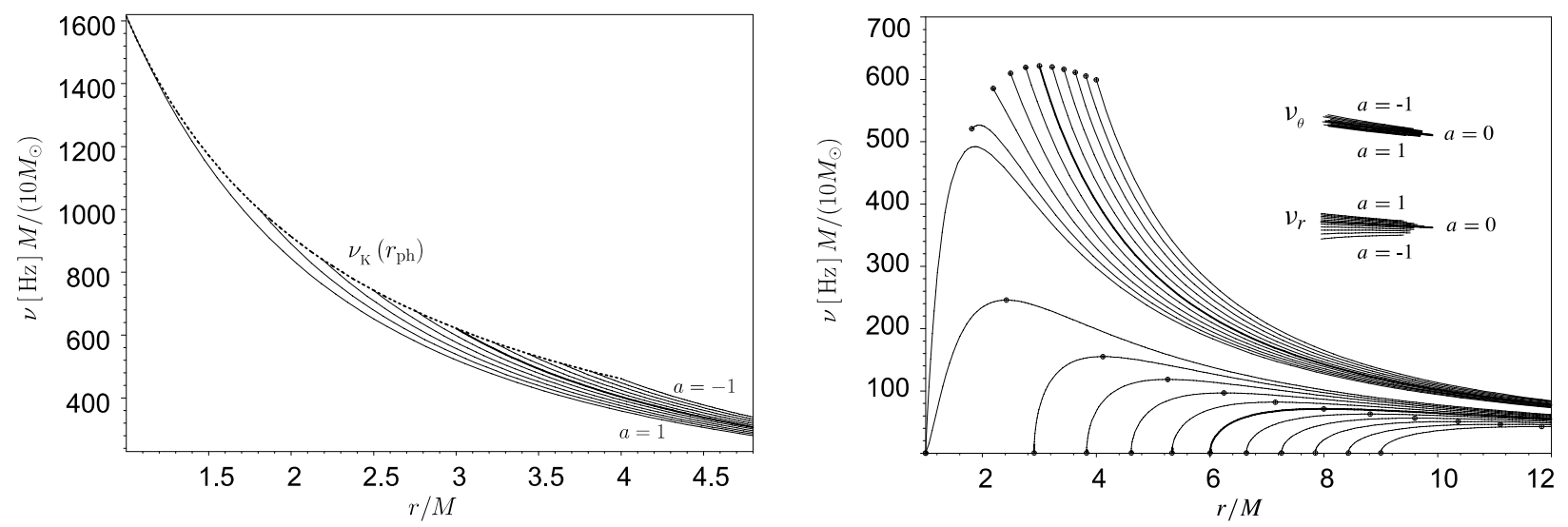

Fig. 1. Keplerian frequency $v_{K}$ (left panel - taken from Abramowicz et al. 2004), and the two epicyclic frequencies (right panel) for Keplerian circular orbits around Kerr black holes. The curves are spaced by 0.2 in $a$.

object angular momentum with $a=J_{0} c / \mathrm{G} M_{0}^{2}$. Here, the parameters $M_{0}$ and $J_{0}$ give the mass and the internal angular momentum of the Kerr black hole or naked singularity.

In the limit of the Schwarzschild black holes $(a=0)$, we arrive at

$\alpha_{\mathrm{r}}(x)=1-\frac{6}{x}$,

$\alpha_{\theta}(x)=1$,

so that $\Omega_{\mathrm{K}}(x)=\omega_{\theta}(x)$.

In the field of Kerr black holes $(a \neq 0)$, there is

$\Omega_{\mathrm{K}}(x, a)>\omega_{\theta}(x, a)>\omega_{\mathrm{r}}(x, a)$

in the range where the frequencies are well defined (Fig. $1-$ right panel).

The properties of $\Omega_{\mathrm{K}}, \omega_{\mathrm{r}}, \omega_{\theta}$ for Kerr black-hole spacetimes are rewieved, e.g., in Kato et al. (1998). We can summarize that

- the Keplerian frequency is a monotonically decreasing function of radius for the whole range of black hole rotational parameter $a \in(-1,1)^{2}$ in astrophysically relevant radii above the photon orbit (Fig. 1 - left panel)

- for slowly rotating black holes the vertical epicyclic frequency is a monotonically decreasing function of radius in the same radial range as well; however, for rapidly rotating black holes this function has a maximum (Fig. 1 - right panel)

- the radial epicyclic frequency has a local maximum for all $a \in(-1,1)$ (Fig. 1 - right panel)

For Kerr naked singularities the behaviour of the epicyclic frequencies is different. In the next sections we show that the vertical frequency can have two local extrema, and the radial one even three. For completeness, we shall discuss the properties of the functions $\Omega_{\mathrm{K}}(x, a), \omega_{\mathrm{r}}(x, a)$, and $\omega_{\theta}(x, a)$ for both naked singularities and black holes.

Obviously, all three frequencies (1)-(3) have the general form,

$v=\left(\frac{G M_{0}}{r_{G}^{3}}\right)^{1 / 2} f(x, a) \doteq 32.3\left(\frac{M_{0}}{M_{\odot}}\right) f(x, a) \mathrm{kHz}$

${ }^{2}$ Here and henceforth values of $a>0$ correspond to corotating orbits, while $a<0$ give counterrotating orbits.
Thus for the reader's convenience we express the frequency as $v[\mathrm{~Hz}] M /\left(10 M_{\odot}\right)$ in every quantitative plot of frequency dependence on radial coordinate (6); i.e., displayed value is the frequency relevant for a central object with a mass of $10 M_{\odot}$, which could be simply rescaled for another mass by just dividing the displayed value by the respective mass in units of ten solar mass.

\section{Properties of the epicyclic frequencies}

First, it is important to find the range of relevance for the functions $\Omega_{\mathrm{K}}(x, a), \omega_{\mathrm{r}}(x, a)$, and $\omega_{\theta}(x, a)$ above the event horizon located at

$x_{+}=1+\sqrt{1-a^{2}}$

for black holes, and above the ring singularity located at

$x=0 \quad(\theta=\pi / 2)$

for naked singularities.

The circular geodesics in the field of Kerr black holes were discussed in Bardeen et al. (1972), while in the case of Kerr naked singularities the circular geodesics were discussed in Stuchlík (1980). We can summarize that circular geodesics can exist in the range of

$x \in\left(x_{\mathrm{ph}}(a), \infty\right)$,

where

$x_{\mathrm{ph}}(a)=2\left[1+\cos \left(\frac{2}{3} \arccos (-a)\right)\right]$

gives loci of photon circular geodesics. Stable circular geodesics, relevant for the Keplerian, thin accretion discs exist in the range of

$x \in\left(x_{\mathrm{ms}}(a), \infty\right)$,

where $x_{\mathrm{ms}}(a)$ denotes the radius of the marginally stable orbit, determined (in an implicit form) by the relation

$1-6 x^{-1}+8 a x^{-3 / 2}-3 a^{2} x^{-2}=0$, 

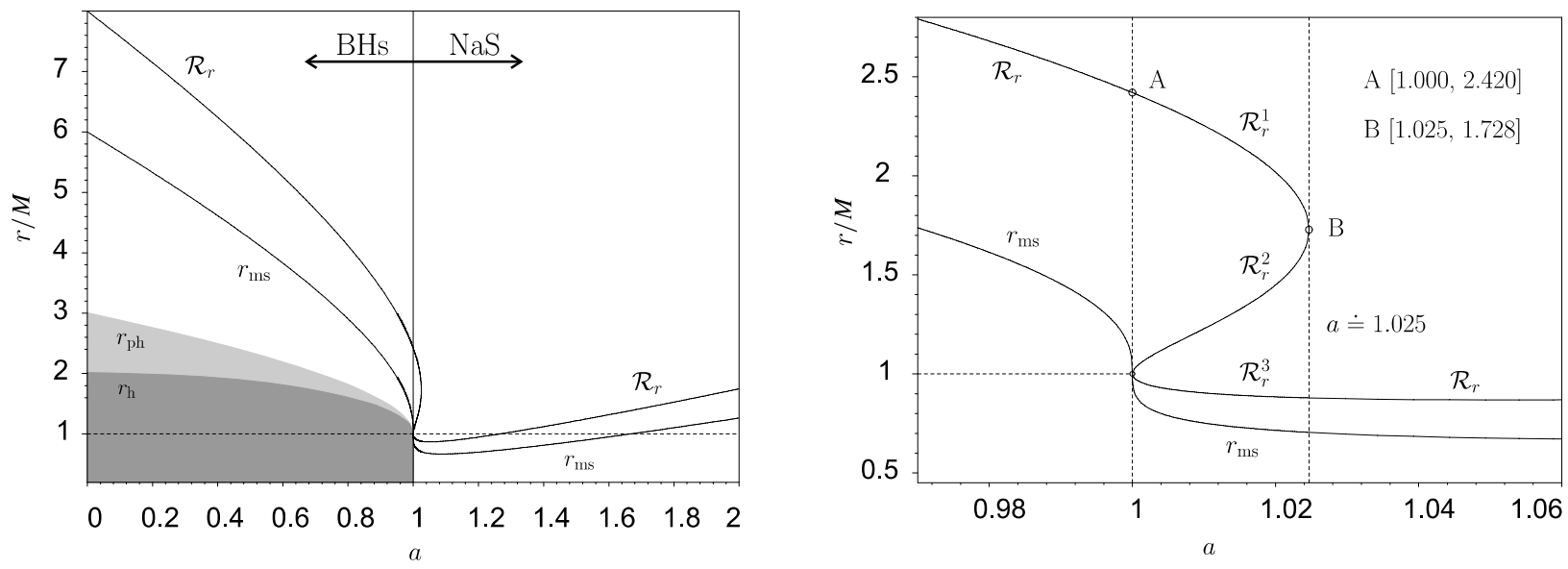

Fig. 2. The locations $\mathcal{R}_{\mathrm{r}}^{i}$ of the radial epicyclic frequency local extrema. Right panel: detailed view. Here, in the next figure and henceforth we use the following convention for both kinds of extrema of the radial $\left(\mathcal{R}_{\mathrm{r}}^{i}\right)$ and the vertical $\left(\mathcal{R}_{\theta}^{i}\right)$ epicyclic frequencies: odd or missing superscript denotes a local maximum and even-numbered one means a local minimum. The question whether in the case of naked singularities the maximum is global one can be addressed by the left panel in Fig. 8 .

which coincides with the condition

$\alpha_{\mathrm{r}}(x, a)=0$.

For toroidal, thick accretion discs the unstable circular geodesics can be relevant in the range

$x_{\mathrm{mb}} \leq x_{\mathrm{in}}<x<x_{\mathrm{ms}}$,

being stabilized by pressure gradients in the tori. Here,

$x_{\mathrm{mb}}=2-a+2 \sqrt{1-a}$

is the radius of the marginally bound circular geodesic that is the lower limit for the inner edge of thick discs (Kozłowski et al. 1978; Krolik \& Hawley 2002).

Clearly, the Keplerian orbital frequency is well defined up to $x=x_{\mathrm{ph}}(a)$. However, $\omega_{\mathrm{r}}$ is well defined, if $\alpha_{\mathrm{r}} \geq 0$, i.e., at $x \geq x_{\mathrm{ms}}(a)$, and $\omega_{\mathrm{r}}(x)=0$ at $x_{\mathrm{ms}}$. We can also show that for $x \geq x_{\mathrm{ph}}$, there is $\alpha_{\theta} \geq 0$; i.e., the vertical frequency $\omega_{\theta}$ is well defined at $x>x_{\text {ph }}$.

\subsection{Local extrema of epicyclic frequencies}

Denoting by $\mathcal{R}_{\mathrm{K}}, \mathcal{R}_{\mathrm{r}}, \mathcal{R}_{\theta}$ the local extrema of Keplerian $v_{\mathrm{K}}$ and epicyclic $v_{\mathrm{r}}, v_{\theta}$ frequencies, we can give the extrema by the condition

$\frac{\partial}{\partial r} v_{i}=0 \Leftrightarrow \frac{\partial}{\partial x} v_{i}=0$ for $\mathcal{R}_{i}, \quad i \in\{\mathrm{K}, r, \theta\}$,

where $x$ is dimensionless coordinate (6). From (1)-(3), we find that the corresponding derivatives ${ }^{3}$ are

$\Omega_{\mathrm{K}}^{\prime}=-\frac{3}{2} \sqrt{\frac{G M_{0}}{r_{G}^{3}}} \frac{\sqrt{x}}{\left(x^{3 / 2}+a\right)^{2}}$,

$\omega_{j}^{\prime}=\frac{3}{2}\left[\frac{2 \beta_{j}}{\sqrt{\alpha}_{j}}-\frac{\sqrt{\alpha_{j} x}}{\left(x^{3 / 2}+a\right)}\right] \Omega_{\mathrm{K}}$,

$\alpha_{j}^{\prime}=6 \beta_{j} / \alpha_{j}$,

\footnotetext{
${ }^{3}$ After introducing' as $\mathrm{d} / \mathrm{d} r$.
}

where $j \in\{r, \theta\}, \quad$ and

$\beta_{\mathrm{r}}(x, a)=\frac{1}{x^{2}}-2 \frac{a}{x^{5 / 2}}+\frac{a^{2}}{x^{3}}$,

$\beta_{\theta}(x, a)=\frac{a}{x^{5 / 2}}-\frac{a^{2}}{x^{3}}$.

Clearly, $\Omega_{\mathrm{K}}^{\prime}<0$ for $x>0$; i.e., the Keplerian frequency is a monotonically decreasing function of the radial coordinate for any value of the rotational parameter $a$.

Relations (20) and (22) imply the condition determining extrema $\mathcal{R}_{j}(a)$ of the epicyclic frequencies:

$\beta_{j}(x, a)=\frac{1}{2} \frac{\sqrt{x}}{x^{3 / 2}+a} \alpha_{j}(x, a) \quad j \in\{r, \theta\}$.

Because we have checked that in the case of counterrotating orbits $(a<0)$ the extrema $\mathcal{R}_{\theta}$ are located under the photon circular orbit and the extrema $\mathcal{R}_{\mathrm{r}}$ are just extensions of the $\mathcal{R}_{\mathrm{r}}$ for corotating case $(a<0)$, we focuse mainly on the case of corotating orbits in the next discussion. In Figs. 2 (3) we show curves $\mathcal{R}_{\mathrm{r}}^{k}(a), k \in\{1,2,3\}\left(\mathcal{R}_{\theta}^{l}(a), l \in\{1,2\}\right)$ implicitly determined by the relations (26); indexes $k, l$ denote different branches of the solution of (26). The radial epicyclic frequency has one local maximum for Kerr black holes

$-1 \leq a \leq 1$,

but it has two local maxima ${ }^{4}$ and one local minimum for Kerr naked singularities with

$1<a<a_{\mathrm{c}(r)} \doteq 1.025$,

and again one local maximum for

$a \geq a_{\mathrm{c}(r)}$ and $a<-1$.

The vertical epicyclic frequency has a local maximum at $x>x_{\mathrm{ph}}$ for Kerr black holes with

$a>a_{\mathrm{ph}(\theta)} \doteq 0.748$,

${ }^{4}$ We distinguish which is the global one in Sect. 4. 

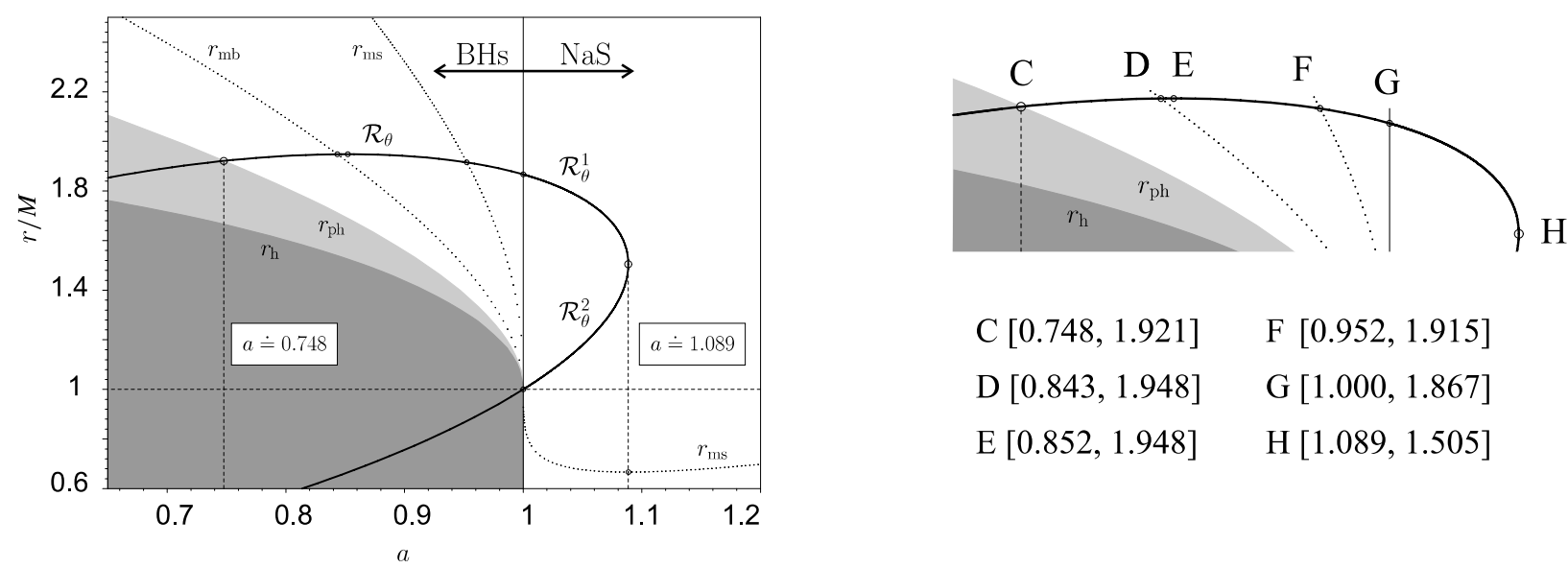

$$
\begin{array}{ll}
\text { C }[0.748,1.921] & \text { F }[0.952,1.915] \\
\text { D }[0.843,1.948] & \text { G }[1.000,1.867] \\
\text { E }[0.852,1.948] & \text { H }[1.089,1.505]
\end{array}
$$

Fig. 3. The locations $\mathcal{R}_{\theta}^{i}$ of the vertical epicyclic frequency local extrema. The right panel gives exact information about the positions of important points.

and at $x>x_{\mathrm{ms}}$ for

$a>a_{\mathrm{ms}(\theta)} \doteq 0.952$.

The local maximum of $\omega_{\theta}(x, a)$ is relevant in resonant effects for $a>a_{\mathrm{ms}(\theta)}$. Note that $\mathcal{R}_{\theta}$ has a maximum at

$a_{\max \left(\mathcal{R}_{\theta}\right)} \doteq 0.852 ;$

therefore, the situation with function $\omega_{\theta}(x, a)$ is more complicated than seems to be indicated in Fig. 1: for high values of the black hole rotational parameter $a$, curves $\omega_{\theta}(x, a)$ cross each other as is shown in the left panel of Fig. 4, while Fig. 1 does not show such detail because of hi-spacing between curves. In the Kerr naked singularity spacetimes, the function $\omega_{\theta}(x, a)$ has a local minimum and a local maximum for

$1<a<a_{\mathrm{c}_{\theta}} \doteq 1.089$,

and has no astrophysically relevant local extrema for

$a \geq a_{\mathrm{c}_{\theta}} \quad$ and $\quad a<-1$.

Using properties of $\mathcal{R}_{\mathrm{r}}(a)$ and $\mathcal{R}_{\theta}(a)$, we can conclude that two qualitatively different types of behaviour exist for the epicyclic frequencies in the Kerr black-hole spacetimes along with three qualitatively different types of their behaviour in the Kerr naked-singularity spacetimes. Examples of the behaviour of the epicyclic frequencies for Kerr black holes are given in Fig. 1 (see also Fig. A.1).

An example of the behaviour of the epicyclic frequencies in Kerr naked-singularity spacetimes is shown in Fig. 4 (right panel) for the case when all the local extrema mentioned above are present, while for an example of the case when the number of the local extrema is lowest see Fig. 9. The complete set of figures systematically representing the evolution of the character of the epicyclic frequencies with rotational parameter increasing is included in the Appendix which consists of Figs. A.1 (black holes) and A.2 (naked singularities); the evolution of derivatives (22) and of the ratio $v_{\theta} / v_{\mathrm{r}}$ of the epicyclic frequencies is also included. This set of figures represents classification of the Kerr spacetimes according to the properties of the epicyclic frequencies fully given in Sect. 5. Note that in the black-hole case it is important to distinguish the cases when the local maximum of $v_{\theta}(x, a)$ is located above $x_{\mathrm{ms}}$, and under $x_{\mathrm{ms}}$.

Clearly, the behaviour of the epicyclic frequencies substantially differs for Kerr naked singularities in comparison with Kerr black holes. We discuss some consequences of this different behaviour for naked singularities in Sect. 4, but first focus our attention on some properties of the epicyclic frequencies that are very important for treating the resonant oscillation phenomena from the observational point of view.

\subsection{Ratio of epicyclic frequencies}

The ratio of epicyclic frequencies $v_{\theta}$ and $v_{\mathrm{r}}$ needs to be defined well for some models of QPOs (e.g., Abramowicz et al. 2004; Kato 2004a). It is well known (see, e.g., Kato et al. 1998) that for the Kerr black holes $(-1 \leq a \leq 1)$ the inequality

$\omega_{\mathrm{r}}(x, a)<\omega_{\theta}(x, a)$

holds, i.e., the equation

$\omega_{\mathrm{r}}(x, a)=\omega_{\theta}(x, a)$

does not have any real solution in the whole range of black hole rotational parameter $a \in(-1,1)$ and

$\left(\frac{v_{\theta}}{v_{\mathrm{r}}}\right)>1$

for any Kerr black hole. Furthermore, this ratio is a monotonic function of radius for any fixed $a \in(-1,1)$ (see Fig. 5 - left panel). However, the situation is different for Kerr naked singularities, see Sect. 4.2.

\subsection{Implications for the orbital resonance models in the field of Kerr black holes}

The orbital resonance models for QPOs proposed by Abramowicz \& Kluzniak (Abramowicz \& Kluźniak 2001; Abramowicz et al. 2004) are particularly based on resonance between epicyclic frequencies which are excited at a well defined resonance radius $r_{p: q}$ given by the condition

$\frac{\omega_{\theta}}{\omega_{\mathrm{r}}}\left(a, r_{p: q}\right)=\frac{p}{q}$ 

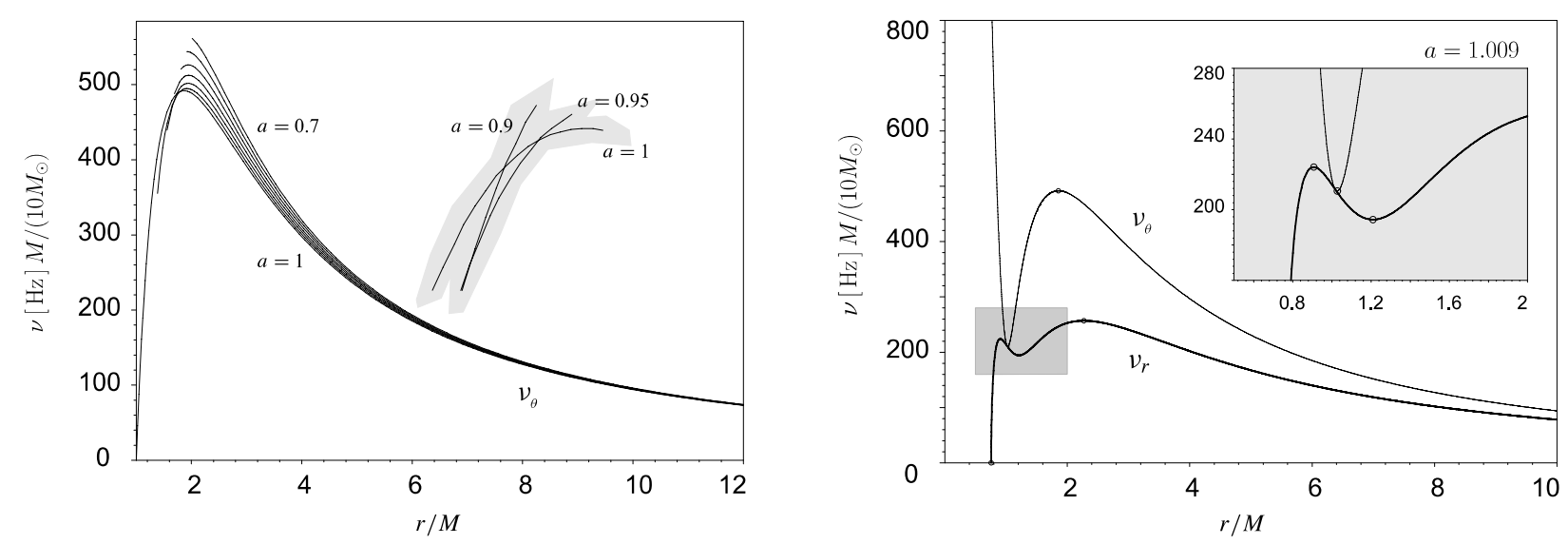

Fig. 4. Left panel: "unlikely" effects resulting from the existence maxima of $\mathcal{R}_{\theta}$ (the point E in Fig. 3). Curves $v_{\theta}(r)$ after $a \doteq 0.852$ cross each other (curves differ in rotational parameter here by 0.05 ), see also Fig. 1 for comparison. The right panel displays an example of epicyclic frequency behaviour for Kerr naked singularity with $a=1.009$; all allowed extrema are present. Note that the minimum $v_{\theta}$ is very close but not identical to the point of contact, which is also present (see Sect. 4.2 for details).
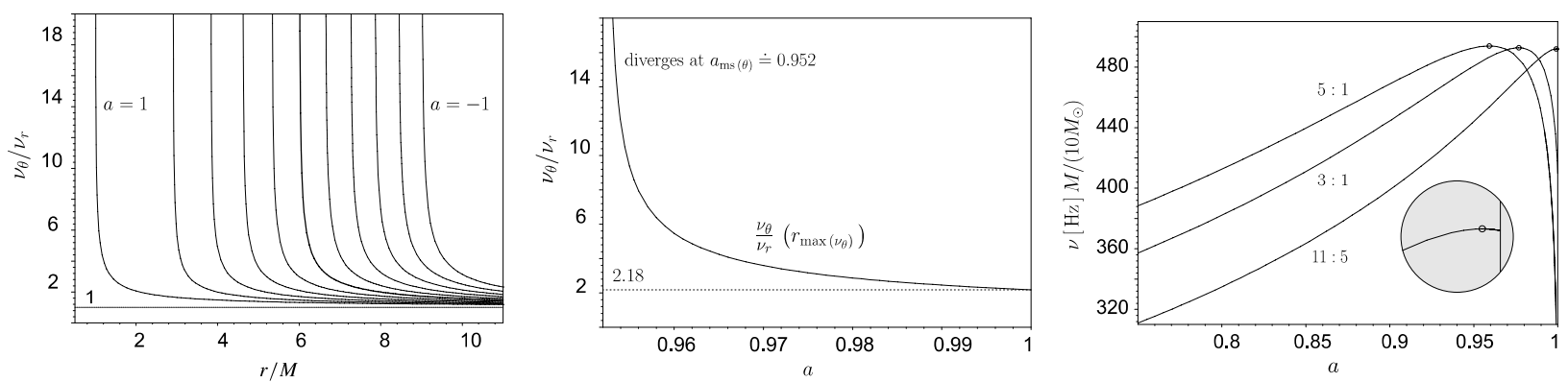

Fig. 5. Left panel: the behaviour of ratio $v_{\theta} / v_{\mathrm{r}}$ of the epicyclic frequencies, with curves spaced by 0.2 in rotational parameter. The middle panel gives information about the ratio between epicyclic frequencies at maxima of $v_{\theta}$, while the right panel illustrates the examples of the behaviour of frequency $v_{\theta}$ for three cases of forced resonances with $p / q=5,3$, and 2.2.

where $p: q$ is $3: 2$ in the case of parametric resonance and arbitrary rational ratio of two small integral numbers $(1,2,3 \ldots)$ in the case of forced resonances. Notice that in the case of arbitrary forced resonance the combinational ("beat") frequencies could also be observed including the 3:2 ratio (Abramowicz \& Kluźniak 2001; Török et al. 2005). Such resonance radii are monotonically decreasing functions of the rotational parametr $a$ (see Fig. 5 - left panel). Resulting resonant frequencies are given generally as a linear combination of epicyclic frequencies at $r_{p: q}$. In Török et al. (2005) it is reported that the resonant frequencies (both observed frequencies, the upper and the lower) are not monotonic function of $a$ for the case of 3:1 and $5: 1(5: 2)$ forced resonance models, while for other resonance model ratios (e.g., 2:1, 3:2) discussed in Török et al. (2005), it is a monotonic function of the rotational parameter $a$. For the observational consequences, it is important to determine the limiting value of the frequency ratios $\omega_{\theta} / \omega_{\mathrm{r}}=p / q$, which separates the monotonic and nonmonotonic dependence of the resonant frequencies on the rotational parameter.

Indeed, this monotonicity of some resonant frequencies results from the nonmonotonic character of the epicyclic frequencies. It is known that the radial epicyclic frequency has a local maximum at $r_{\max (\mathrm{r})} \equiv \mathcal{R}_{\mathrm{r}}$ for $a \in(-1,1)$ and its value $v_{\mathrm{r}(\max )}(a)$ increases with the rotational parameter (see Fig. 1 right panel). Moreover, outside its maxima it is monotonically decreasing with the radius. From the left panel of Fig. 5 we conclude that $r_{p: q}$ (a) must be a monotonically decreasing function of $a$. If the horizontal line representing some ratio $p: q$ is fixed, then this figure implies a necessarily monotonically decreasing function $r_{p: q}(a)$. Because of this, the resulting resonant frequency, which is just multiple of the radial frequency, must be monotonically increasing for $r_{p: q}$ located outside (or at) the maximum of the radial epicyclic frequency. For Schwarzschild black holes the ratio between the epicyclic frequencies at the radius of maximal radial frequency is exactly $v_{\theta} / v_{\mathrm{r}}=2(x=8)$ and then changes slighty with the rotational parameter growing to reach the value $v_{\theta} / v_{\mathrm{r}} \sim 1.8$ for extremely rotating Kerr black hole $(a=1)$. This gives the limit in the sense that for $p: q>1.8$, the radius $r_{p: q}$ is certainly located above the maximum of $v_{\mathrm{r}}$.

On the other hand, an analogical consideration shows that $v_{\theta}$ (or $v_{\mathrm{r}}$, if the resonance condition (38) is satisfied) is surely decreasing with the rotational parameter, if $r_{p: q}$ is located under the location of the maximum of $v_{\theta}$. The ratio of the epicyclic frequencies at the maximum of $v_{\theta}$ is shown in the middle panel of Fig. 5. Its minimum is reached for extremely rotating Kerr black holes at $v_{\theta} / v_{\mathrm{r}}\left(r_{\max }\right) \sim 2.18$. It means that for black holes the resonance is surely nonmonotonic if $p / q \geq 2.18$.

It is clear from the discussion above that the limit for nonmonotonicity must be located between the values of $p / q \in$ $(1.8,2.18)$. We numerically checked the loci of eventual 

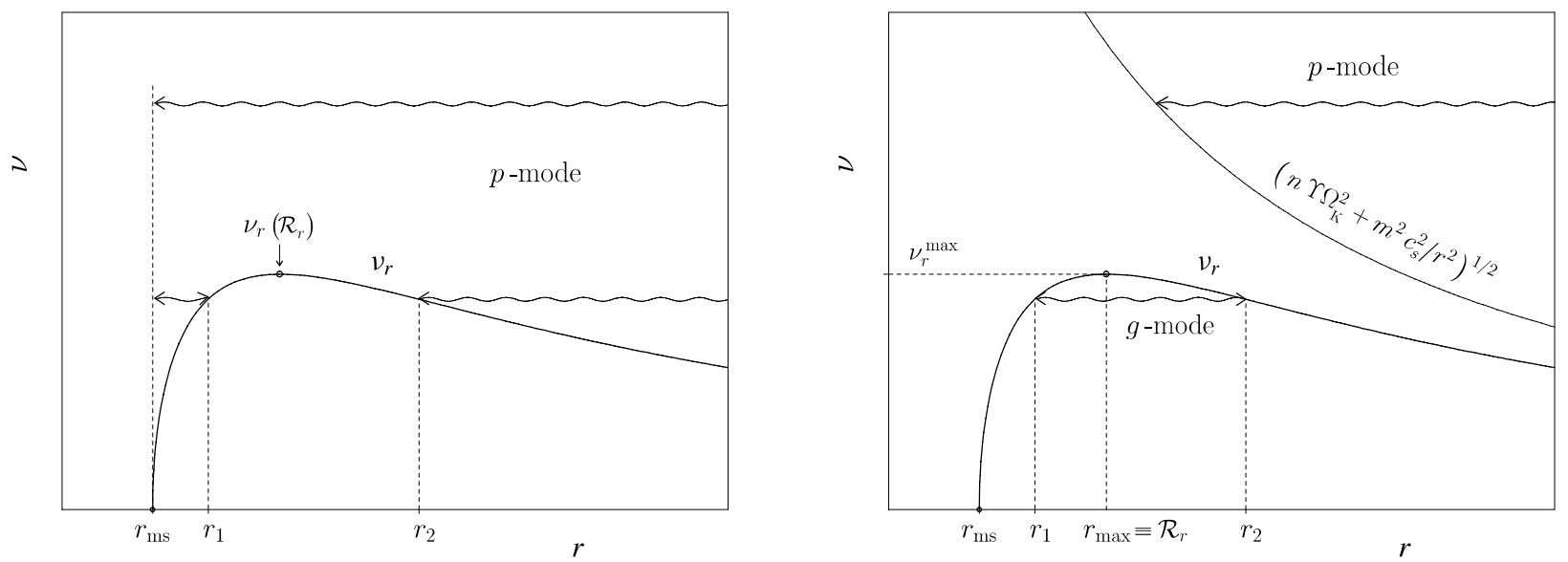

Fig. 6. (After Kato et al. 1998) The simplest examples of wave propagation in a black-hole thin accretion disc: for fundamental axisymmetric mode $(n=m=0)$ see left panel, while the first axisymmetric overtone $(n=1, m=0)$ is shown in the right one. Both figures are plotted for the Schwarzschild black hole.

nonmonotonicity and find that the limit is very close to the upper value; i.e., nonmonotonicity of the function $v_{\theta} / v_{\mathrm{r}}\left(r_{p: q}, a\right)$ in dependence on $a$ is relevant for forced resonances with

$p: q \geq 2.18$.

Figure 5 (right panel) illustrates this limit by examples of behaviour of $v_{\theta}\left(a, r_{p: q}\right)$ for three different forced resonances, which embody the nonmonotonicy (in the sense described above).

\section{Some important implications for the discoseismology of Kerr naked singularities}

Without going into detail, we explore simple examples to show that the physics of wave propagation and oscillations in thin accretion discs around the Kerr naked singularities is considerably different from the Kerr black hole case.

\subsection{Modified propagation of inertial-acoustic and inertial-gravity waves}

The systematic classification of oscillations and waves in accretion discs according to the restoring forces acting on them was summarized, e.g., in Kato et al. (1998). They introduced the following modes for disc oscillations:

- The higher frequency modes, i.e., inertial-acoustic waves, so-called $p$-modes.

- The lower frequency modes, i.e., inertial-gravity waves, socalled $g$-modes.

- The inertial-acoustic modes with no node in vertical direction are called corrugation waves.

We recall that the wave propagation is given by the dispersion relation (Okazaki et al. 1987; Kato 1989; Nowak \& Wagoner 1991, 1992)

$\left(\omega^{2}-\omega_{\mathrm{r}}^{2}\right)\left(\omega^{2}-n \Upsilon \Omega_{\mathrm{K}}^{2}-\frac{m^{2} c_{\mathrm{s}}^{2}}{r^{2}}\right)=\omega^{2} k_{\mathrm{r}}^{2} c_{\mathrm{s}}^{2}$, where $\omega$ is the angular velocity corresponding to the frequency of the propagated wave, $\Upsilon$ a correction factor of the order of unity, $k_{\mathrm{r}}$ the wave vector, and $c_{\mathrm{s}}$ the vertically averaged sound speed. For the case of axially symmetric $(m=0)$ fundamental mode $(n=0)$ this relation is reduced to

$\omega^{2}=\omega_{\mathrm{r}}^{2}+k_{\mathrm{r}}^{2} c_{\mathrm{s}}^{2}$.

In this simple case the only waves of $p$-mode $\left(\omega^{2}>\omega_{\mathrm{r}}^{2}\right)$ can propagate in the radial direction and could be in the black hole case separated into two groups (Kato et al. 1998):

- The waves with ${ }^{5} \omega^{2}>\omega_{\max }^{2}$ can propagate through the entire region of the disc.

- For the waves with $\omega^{2}<\omega_{\max }^{2}$ the propagation region is separated into two discrete portions (see Fig. 6 - left panel):

- Inner region $r<r_{1}$.

- Outer region $r>r_{2}$,

where $r_{1}, r_{2}$ are the solutions of $\omega^{2}=\omega_{\mathrm{r}}^{2} ; \pi$-waves with $\omega^{2}<\omega_{\max }^{2}$ cannot propagate between $r_{1}$ and $r_{2}$, however they can outside this region. Because of the physical properties of the inner edge of the disc, the $\pi$ waves could be trapped in the inner region; i.e., they are reflected between the inner edge and $r_{1}$ as shown by Kato \& Fukue (1980).

The overtones $(n \neq 0)$ in Eq. (40) allow for the inertial acoustic $p$-waves, as well as inertial gravity $g$-waves. Generally, $p$-waves in this case must have frequency $\omega^{2}>n \Upsilon \Omega_{\mathrm{K}}^{2}$, while for $g$-waves the inequality $\omega^{2}<\omega_{\mathrm{r}}^{2}$ must hold. The $g$-waves that could be trapped (see Fig. 6 - right panel) in the region around $r_{\max (\mathrm{r})} \equiv \mathcal{R}_{\mathrm{r}}$ are insensitive to changes in disc structure, if the condition of a moderately geometrically thin disc is satisfied (Okazaki et al. 1987; Nowak \& Wagoner 1992; Perez et al. 1997).

In the case of Kerr naked singularities, the well-known picture of the wave propagation, briefly recalled above, is changed due to the different behaviour of the epicyclic frequencies.

\footnotetext{
${ }^{5}$ Here and henceforth in relation to the wave propagation in the field of black holes $\omega_{\max }$ denotes the maximum of $\omega_{\mathrm{r}}$ at $\mathcal{R}_{\mathrm{r}}(a)$ (Fig. 2) in the black-hole range of the rotational parameter.
} 

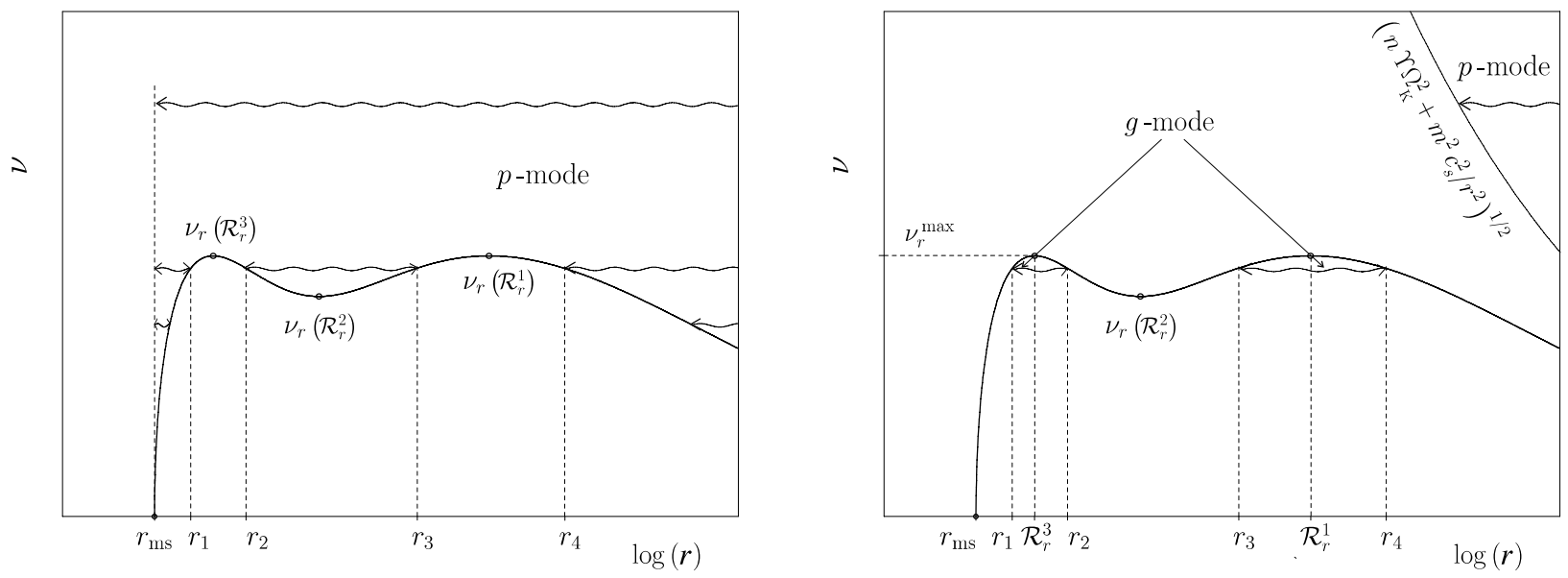

Fig. 7. The same examples of wave propagation as in Fig. 6, but for naked-singularity thin accretion disc (for the simplicity in degenerated case of $a \doteq 1.012$ ). Fundamental axisymmetric modes appear in the left panel and the first axisymmetric overtone in the right one.

As shown in Sect. 3, the radial epicyclic frequency has two global maxima for Kerr naked singularities with $1<a<a_{\mathrm{c} r} \doteq 1.025$. Thus propagation of the fundamental axisymmetric ( $n=0, m=0) \pi$-waves is changed (of course, the condition $\omega^{2}>\omega_{\mathrm{r}}^{2}$ must be satisfied again):

- The waves with ${ }^{6} \omega^{2}>\omega_{\text {MAX }}^{2}$ can propagate through the entire region of the disc, as well as in the black hole case.

- The waves with $\omega^{2}<\omega_{\text {MAX }}^{2}$ could be divided into three groups

(i) For waves with $\omega>\omega_{\max }$ the propagation region splits into two (inner and outer) regions as in the case of black holes.

(ii) For waves with $\omega_{\min }<\omega<\omega_{\max }$ the propagation region splits into three different portions from which two could contain trapped waves.

(iii) For waves with $\omega_{\min }>\omega$ the propagation region splits again into inner and outer region as in the case of a black hole.

This modified wave propagation is illustrated in Fig. 7 (left panel) for the simple degenerated case $a \doteq 1.012$ when $\omega_{\max }=\omega_{\mathrm{MAX}}$. In Fig. 7 (right panel) we illustrate that analogically to the situation described above for trapped $\pi$-waves of fundamental mode, the propagation of trapped $g$-modes $(n \neq 0)$ splits into discrete portions in the case of naked singularities with $a<a_{\mathrm{c} r} \doteq 1.025$. For the Kerr naked singularities with rotational parameter $a>a_{\mathrm{c} r}$, the radial epicyclic frequency has the same properties as in the black hole case and the wave propagation is formally the same as for black holes.

\subsection{Strong resonant frequency}

It is shown in Sect. 3 that for Kerr naked singularities with $a>a_{\mathrm{c}(\theta)} \doteq 1.089$ the behaviour of epicyclic frequencies is formally similar to Kerr black holes. However, for any naked

\footnotetext{
${ }^{6}$ In the relation to the wave propagation in discs around Kerr naked singularities, $\omega_{\text {MAX }}$ denotes the global maximum of $\omega_{\mathrm{r}}$, while $\omega_{\max }$ denotes a local (lower) one, see Fig. 8.
}

singularity with $a \geq 1$, the epicyclic frequencies $(2,3)$ can satisfy the equality condition

$\omega_{\mathrm{r}}(a, x)=\omega_{\theta}(a, x)$

giving a strong resonant phenomenon ${ }^{7}$, which occurs at the critical radius

$x_{\mathrm{sr}}=a^{2} \quad(a \geq 1)$.

This means that for any Kerr naked singularity the epicyclic frequency ratio $v_{\theta} / v_{\mathrm{r}}(r)$ is a nonmonotonic function that reaches value 1 at the point given by (43) (see Fig. 8 - middle panel). The loci of this point are compared with locations of some other important points as shown in Fig. 9 - the right panel; while the left panel shows an example that illustrates radial extension of the strong resonant phenomenon.

Using the relation $(43)$ in $(2,3)$ we find a strong resonant frequency that, in terms of the corresponding angular velocity, reads

$\omega_{\mathrm{sr}} \equiv \omega_{\mathrm{r}}\left(a, a^{2}\right)=\omega_{\theta}\left(a, a^{2}\right)=\left(\frac{G M_{0}}{r_{G}^{3}}\right)^{1 / 2} \frac{\sqrt{a^{2}-1}}{a^{2}\left(a^{2}+1\right)}$,

and the frequency can be expressed in the form

$v_{\mathrm{sr}}=32.3\left(\frac{M_{\odot}}{M}\right) \frac{\sqrt{a^{2}-1}}{a^{2}\left(1+a^{2}\right)} \mathrm{kHz}$.

We note that this strong-resonance phenomenon represents a crucial difference between Kerr naked singularities and the case of Kerr black holes for which the ratio $\omega_{\theta} / \omega_{\mathrm{r}}(r)$ is determined as a monotonic function for fixed $a$ (Fig. 5 - left panel).

The behaviour of the epicyclic frequency ratio $\omega_{\theta} / \omega_{\mathrm{r}}(r)$ typical of Kerr naked singularities is shown in Fig. 8 (middle panel). In right panel in Fig. 8 we plot the value of the local extrema of the ratio $\omega_{\theta} / \omega_{\mathrm{r}}$ as a function of the rotational parameter $a$. For high values of the rotational parameter, the radial and vertical epicyclic frequencies are very close each other in large radial range around $r_{\mathrm{sr}}$. This example is given in Fig. 9 (left panel). We plot the strong-resonance frequency as a function

\footnotetext{
${ }^{7}$ Intuitively clear attribution is well founded in the last Sect. 4.4.
} 

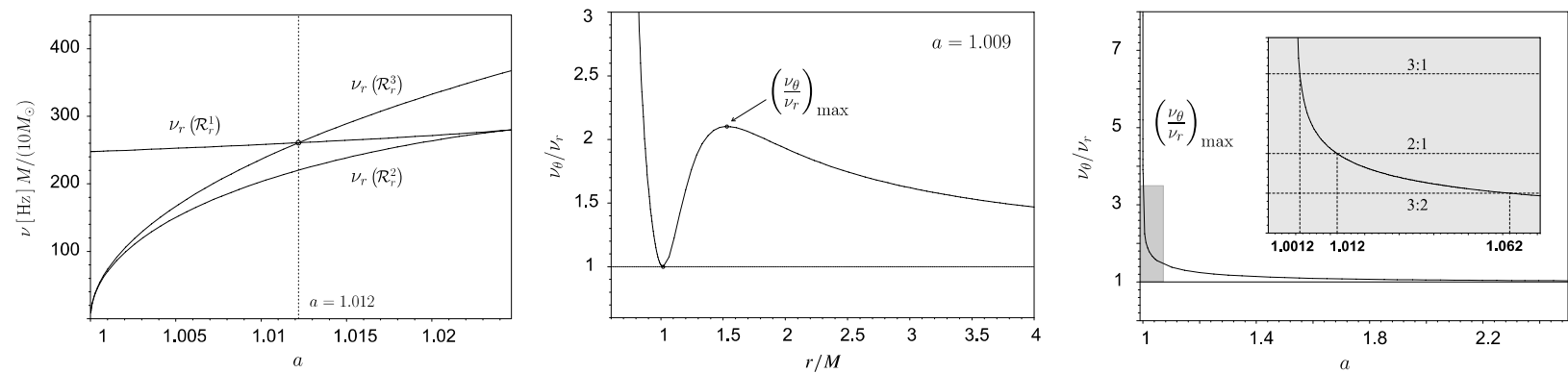

Fig. 8. Left panel: the radial epicyclic frequency at particular extrema as a function of rotational parameter $a$ between $a=1$ and $a=1.025$. We can see that for $a<1.012$ the global maximum is situated at $\mathcal{R}_{\mathrm{r}}^{1}(a)$ while for $a>1.012$ it is at $\mathcal{R}_{\mathrm{r}}^{3}(a)$. The middle panel exposes the behaviour of function $v_{\theta} / v_{\mathrm{r}}(x)$ typical for Kerr naked singularities. With increasing parameter $a$ the local maximum of this function is shifted to higher radii. The right panel illustrates that the value of maximum $v_{\theta} / v_{\mathrm{r}}$ is rapidly decreasing with the rotational parameter growing. The upper limit for unambiguity of a resonance in the case of $p: q=3: 1,2: 1$ and $3: 2$ is denoted.
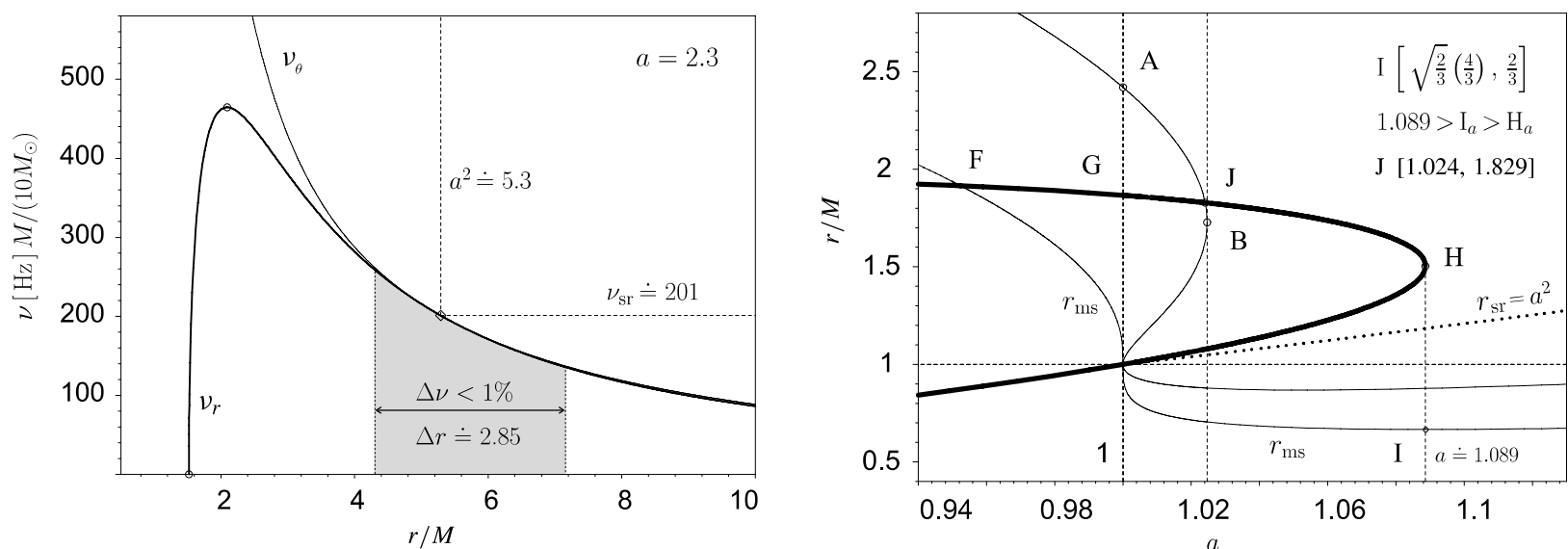

Fig. 9. Left panel: behaviour of epicyclic frequencies for $a=2.3$, the region where frequencies are identical with accuracy of $1 \%$. The right panel illustrates the location of the strong resonant frequency (dotted curve $a^{2}$ ) in relation to extrema of epicyclic frequencies (thin curve $\mathcal{R}_{\mathrm{r}}^{k}$, thick one $\mathcal{R}_{\theta}^{l}$ ). The critical radius is always located (in radial order) between the first maximum of $\omega_{\mathrm{r}}$ and the minimum of $\omega_{\theta}$ (if these exist). The notation of the important points [A... J] accords with Figs. 2 and 3.

of the rotational parameter in Fig. 10 (left panel). It approaches zero value for an extremely rotating Kerr black hole and has a maximum for naked singularities with rotational parameter

$a_{\text {src }} \doteq 1.207$,

with the corresponding value of the epicyclic frequency determined by the relation

$v_{\mathrm{src}} \doteq 6.1\left(\frac{M}{M_{\odot}}\right)^{-1} \mathrm{kHz}$.

In the middle panel of Fig. 10, location of the critical radius $x_{\mathrm{sr}}=a^{2}$ is shown together with location of the marginally stable orbit and extension of the instability region of $r$ (see Sect. 4.4), where the difference between values of the radial and vertical epicyclic frequencies is smaller then $1 \%$. However, for the values of rotational parameter $a \sim 1$, it is more convenient to express the region of the disc with $1 \%$ difference of the epicyclic frequencies in terms of the proper radial distance $\tilde{r}$, which has direct physical meaning. There is

$\tilde{r}=\int_{r_{0}}^{r_{1}} \sqrt{g_{r r}} \mathrm{~d} r$ where $g_{r r}$ denotes the radial metric coefficient of the Kerr metric in the standard Boyer-Lindquist coordinates; the distance is measured from the inner edge of the thin discs located at $r_{\mathrm{ms}}$. The result is represented by the right panel in Fig. 10; we found that the strong-resonance is closest to the inner edge of the Keplerian disc for naked singularity with

$a_{\tilde{\mathrm{r}}} \doteq 1.105$.

For this value of the rotational parameter the strong resonant frequency is

$v_{\text {sr in }} \doteq 4.3\left(\frac{M}{M_{\odot}}\right)^{-1} \mathrm{kHz}$,

which is about $70 \%$ of the maximum at $a_{\text {srmax }}$ given by (47). However, the critical radius is always located outside of the innermost part of the disc (see Fig. 9, right panel).

\subsection{Implication for other resonant effects}

For Kerr naked singularities with any rotational parameter $a$, the ratio $\omega_{\theta} / \omega_{\mathrm{r}}<\left(\omega_{\theta} / \omega_{\mathrm{r}}\right)_{\max }$ being fixed can appear at three different radii. This kind of behaviour results from the existence of the strong resonance frequency $\left(\omega_{\theta}=\omega_{\mathrm{r}}\right)$ for any Kerr 

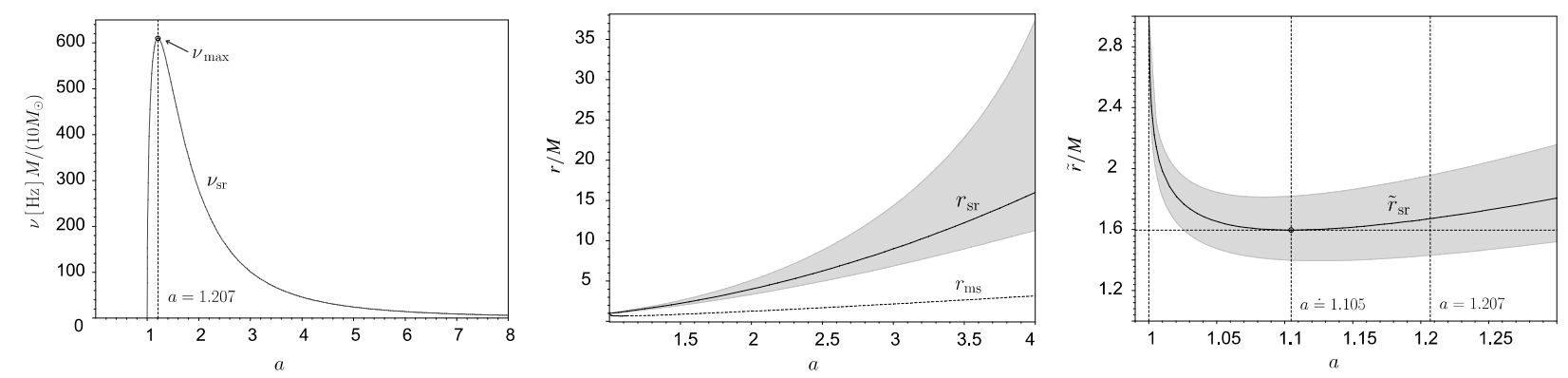

Fig. 10. Left panel: the behaviour of strong resonant frequency shows remarkable maximum for the rotational parameter $a \sim 1.2$. From the middle panel we can see the evolution of area (around critical radius) where epicyclic frequencies are close, while the right panel shows the same in the proper distance to marginally stable orbit.

naked singularity; i.e., it is not restricted to the cases when local extrema of $\omega_{\theta}, \omega_{\mathrm{r}}$ exist. This implies an important consequence for the resonant phenomena: in the case of slowly rotating Kerr naked singularities, an eventual resonance orbit $r_{p: q}$ (with $p, q$ being small integral numbers) is defined ambiguously. In the range of frequencies $\left(\omega_{\theta} / \omega_{\mathrm{r}}\right)_{\max }(a) \geq \omega_{\theta} / \omega_{\mathrm{r}} \geq 1$, the resonant effects with the same rational ratio $p: q$ can occur at three different radii $r_{p: q}$. Using the behaviour of the function $\left(\omega_{\theta} / \omega_{\mathrm{r}}\right)_{\max }(a)$ (Fig. 8 - right panel) we can conclude that three radii $r_{p: q}$ could occur in the field of Kerr naked singularities with $a \leq 1.0012$ for $p: q \leq 3: 1, a \leq 1.012$ for $p: q \leq 2: 1$, and $a \leq 1.062$ for $p: q \leq 3: 2$.

\subsection{Possible instability of the accretion disc around Kerr naked singularities}

The orbital resonance model (Kluźniak \& Abramowicz 2002) demonstrates that fluid accretion flows admit two linear quasiincompressible modes of oscillations, vertical and radial, with corresponding eigenfrequencies equal to vertical and radial epicyclic frequencies for free particles. In a particular model of slender torus, the general properties of these modes can be shown: the vertical mode corresponds to a periodic displacement in which the whole torus moves as a rigid body up and down the equatorial plane and each fluid element has a vertical velocity that periodically changes in time, but does not depend on the position. The frequency of the vertical mode is equal to the vertical epicyclic frequency that a ficticious free particle orbiting at the circle of maximum pressure in the torus equilibrium position would have. Behaviour of the radial mode is similar to the vertical one, and in the linear regime these two modes are formally uncoupled. Kluźniak \& Abramowicz (2002) argue that in the case of more realistic description that includes non-linear effects given by pressure and dissipation, these effects couple the two epicyclic modes that may result in a resonance.

One possible resonance, the parametric resonance, seems to be the most probable explanation of the $3: 2$ double peak $\mathrm{kHz}$ QPOs observed in some galactic microquasars (Abramowicz et al. 2004). The effect itself is described by the Mathieu equation (Landau \& Lifshitz 1976). After denoting the time derivative $\mathrm{d} / \mathrm{d} t$ by dot

$\delta \ddot{\theta}+\omega_{\theta}^{2}\left[1+h \cos \left(\omega_{r} t\right)\right] \delta \theta=0$, which can be formally derived by considering small deviations of fluid streamlines from planar circular motion governed by a set of equations (Rebusco 2004; Horák et al. 2004)

$\delta \ddot{r}+\omega_{r}^{2} \delta r=\omega_{r}^{2} f_{r}(\delta r, \delta \theta, \delta \dot{r}, \delta \dot{\theta})$,

$\delta \ddot{\theta}+\omega_{\theta}^{2} \delta \theta=\omega_{\theta}^{2} f_{\theta}(\delta r, \delta \theta, \delta \dot{r}, \delta \dot{\theta})$,

for a particular choice of $f_{r}$ and $f_{\theta}$, corresponding to

$\delta \ddot{r}+\omega_{r}^{2} \delta r=0, \quad \delta \ddot{\theta}+\omega_{\theta}^{2} \delta \theta=-\omega_{\theta}^{2} \delta \theta \delta r$.

From the theory of the Mathieu equation it is known that the parametric resonance is then excited when ${ }^{8}$

$\frac{\omega_{\mathrm{r}}}{\omega_{\theta}}=\frac{v_{\mathrm{r}}}{v_{\theta}}=\frac{2}{n}, \quad n=1,2,3 \ldots$

The effect is strongest for the smallest possible value of $n$ (Landau \& Lifshitz 1976). Because in the field of black holes $v_{\mathrm{r}}<v_{\theta}$ (see Sect. 3), the smallest possible value for resonance is $n=3$, i.e., $2 v_{\theta}=3 v_{\mathrm{r}}$, which explains the 3:2 ratio observed in microquasars very well (Abramowicz \& Kluźniak 2004; Török et al. 2005).

As shown above, the point where radial epicyclic frequency equals vertical epicyclic frequency exists for any Kerr naked singularity. Obviously, at such a point the equality (54) is satisfied $\left(v_{\mathrm{r}} / v_{\theta}=1 / 1=2 / n ; n=2\right)$, and the parametric resonance (between the radial and vertical epicyclic frequency) eventually excited at this point is the strongest possible parametric resonance excited between the epicyclic frequencies in the field of Kerr naked singularities. Such 2:2 resonance must also be stronger than the 3:2 parametric resonance in the black hole case (Landau \& Lifshitz 1976).

From this, and from the fact that the radial region with epicyclic frequencies that are nearly equal is rather large, one can expect that at this region both radial and vertical oscillations could be strongly amplified, leading to an instability of the accretion $\operatorname{disc}^{9}$.

\footnotetext{
${ }^{8}$ We note that the same condition holds for internal resonance, which describes systems with conserved energy (Horák et al. 2004).

${ }^{9}$ Such a claim is motivated by experience from known situations related to the parametric or forced resonance in complex non-linear systems observed in Earth physics (Landau \& Lifshitz 1976). Examples of mathematically possible resonances causing damaging bridges, wings, etc. with no specific physical coupling mechanism known are discussed in Nayfeh \& Mook (1979).
} 


\section{Conclusions}

For counterrotating Keplerian orbits, properties of the epicyclic frequencies are the same for all Kerr black holes and naked singularities. Radial epicyclic frequency always has a local maximum, while the vertical epicyclic frequency has no local extrema at $x>x_{\mathrm{ph}}$.

On the other hand, for corotating Keplerian orbits, properties of the epicyclic frequencies strongly depend on the rotational parameter of the Kerr spacetimes. The most important difference between spacetimes with $a<1$ and $a>1$ is the change of inequality

$\omega_{\theta}(x)>\omega_{\mathrm{r}}(x) \quad(a<1) \quad \rightarrow \quad \omega_{\theta}(x) \geq \omega_{\mathrm{r}}(x) \quad(a>1)$.

We have also to distinguish different possibilities according to the existence and relative locations of the local extrema of the epicyclic frequencies.

In the case of Kerr black holes, the classification according to the properties of the epicyclic frequencies is given in the following way:

\section{- BH1 (Fig. A.1a) $0<a<0.748$}

$\omega_{\mathrm{r}}(x, a)$ has one local maximum, $\omega_{\theta}(x, a)$ has no local extrema above the photon circular orbit $x_{\mathrm{ph}}$.

- BH2 (Fig. A.1b) $0.748<a<0.952$

$\omega_{\mathrm{r}}(x, a)$ with one local maximum, $\omega_{\theta}(x, a)$ with one local maximum at $x<x_{\mathrm{ms}}$.

- BH3 (Fig. A.1c) $0.952<a<1$

$\omega_{\mathrm{r}}(x, a)$ with one local maximum, $\omega_{\theta}(x, a)$ with one local maximum at $x>x_{\mathrm{ms}}$.

In all the cases, the function $\left(\omega_{\theta} / \omega_{\mathrm{r}}\right)(x, a)$ which is relevant for resonant effects, has a monotonic (descending) character (Fig. A.1). Therefore, for a given rotational parameter $a$, there is only one radius allowed for any $p: q$ resonance. However, we have shown that the resulting resonant frequencies are nonmonotonic functions of $a$ for $p: q>2.18$, which could contradict eventual spin estimate in some resonance models. In addition, curve $\mathcal{R}_{\theta}(a)$ has a local maximum at $a \doteq 0.852$ so we can conclude that the functions $\omega_{\theta}\left(x, a_{1}\right), \omega_{\theta}\left(x, a_{2}\right)$ with $a_{1}, a_{2}$ fixed and higher than $a \simeq 0.85$ cross each other, which can be also of observational interest.

In the case of Kerr naked singularities, the classification is given as follows:

- NaS1 (Fig. A.2a) $1<a<1.012$

$\omega_{\mathrm{r}}(x, a)$ has two local maxima and one local minimum between the maxima;

$\omega_{\mathrm{r}(\max )}\left(x_{\mathrm{r}(\mathrm{in})}, a\right)<\omega_{\mathrm{r}(\max )}\left(x_{\mathrm{r}(\mathrm{out})}, a\right)<\omega_{\theta(\max )}$, where $x_{\mathrm{r}(\mathrm{in})} \equiv \mathcal{R}_{\mathrm{r}}^{3}, x_{\mathrm{r} \text { (out) }} \equiv \mathcal{R}_{\mathrm{r}}^{1}$.

$\omega_{\theta}(x, a)$ has one local minimum and one local maximum.

There is $x_{\theta \text { (max) }} \equiv \mathcal{R}_{\theta}^{1}<x_{\mathrm{r} \text { (out) }}$.

- NaS2 (Fig. A.2b) $1.012<a<1.024$

$\omega_{\mathrm{r}}(x, a)$ has two local maxima and a local minimum in between,

$\omega_{\mathrm{r}(\max )}\left(x_{\mathrm{r}(\mathrm{out})}, a\right)<\omega_{\mathrm{r}(\max )}\left(x_{\mathrm{r}(\mathrm{in})}, a\right)<\omega_{\theta(\max )}$.

$\omega_{\theta}(x, a)$ has one local minimum and one local maximum,

with $x_{\theta(\max )}<x_{\mathrm{r}(\text { out })}$.
- NaS3 (Fig. A.2c) $1.024<a<1.025$

The same as in the class NaS3, but with $x_{\theta \text { (max) }}>x_{\mathrm{r}(\text { out })}$.

- NaS4 $1.025<a<1.047$

$\omega_{\mathrm{r}}(x, a)$ with one local maximum. $\omega_{\theta}(x, a)$ with one local minimum and one local maximum; $\omega_{\theta(\max )} \geq \omega_{\mathrm{r}(\max )}$.

- NaS5 (Fig. A.2d) $1.047<a<1.089$

The same as class NS4, but with $\omega_{\theta(\max )}<\omega_{\mathrm{r}(\max )}$.

- NaS6 (Fig. A.2e) $\quad a>1.089$

$\omega_{\mathrm{r}}(x, a)$ with one local maximum, $\omega_{\theta}(x, a)$ with no local extrema. This class is formally similar to the class $\mathrm{BH} 1$, but with the crucial exception of the point $x_{\mathrm{sr}}$.

We conclude that the properties of the radial and vertical epicyclic frequencies of the Keplerian motion in the case of Kerr naked singularities differ substantially from the case of Kerr black holes, which can have strong observational consequences for both resonant phenomena and the stability of accretion discs around Kerr naked singularities.

While wave propagation in the case of oscillations in the discs around Kerr naked singularities is substantially different from the black hole case only for spacetimes with $a \in$ $(1,1.025)$, the strong resonance effect can occur for any Kerr naked singularity. The strong resonant frequency of disc oscillations around Kerr naked singularities always arise at the descending part of the function $\omega_{\mathrm{r}}(x, a)$, in the vicinity of the local minimum of $\omega_{\theta}$, if this exists; i.e., it is always located above the innermost part of the disc. We stress that this phenomenon represents the strongest parametric resonance between the epicyclic frequencies possible in the field of Kerr naked singularities, stronger than in the case of Kerr black holes. Moreover, the area where the effect occurs is large which could have a strong influence on the stability of the Keplerian disc itself.

It follows from the existence of the strong resonant frequency that the function $\left(\omega_{\theta} / \omega_{\mathrm{r}}\right)(x, a)$ has the same character for all Kerr naked singularities with one local maximum $\left(\omega_{\theta} / \omega_{\mathrm{r}}\right)_{\max }>1$, and one local minimum at $\left(\omega_{\theta} / \omega_{\mathrm{r}}\right)_{\min }\left(x_{\mathrm{sr}}=a^{2}, a\right)=1$ which corresponds to the strong resonant frequency. Because of this, some resonant effects can occur at three different radii $r_{p: q}$ with the same rational ratio $p: q$; i.e., resonant effects with the same ratios could be induced by very different physical phenomena at different parts of the accretion disc.

Finally we stress that the strong resonant frequency represents the phenomenon which we searched for in particulari.e., the effect which could clearly indicate whether an observed $\mathrm{X}$-ray is emitted from the accretion disc orbiting a naked singularity. If the resonant explanation of black-hole double peak QPOs is right, for naked singularities one can expect that the strong resonance 1:1 should significantly modulate in X-ray spectra one unique peak instead of two different peaks.

Acknowledgements. We thank Prof. Marek Abramowicz and Jiří Horák for discussions. This work was supported by the Czech grant MSM 4781305903. We also thank the perfect hospitality of Nordita (Copenhagen). 


\section{Appendix A: Classification of the Kerr spacetimes (due to epicyclic frequencies)}

a)

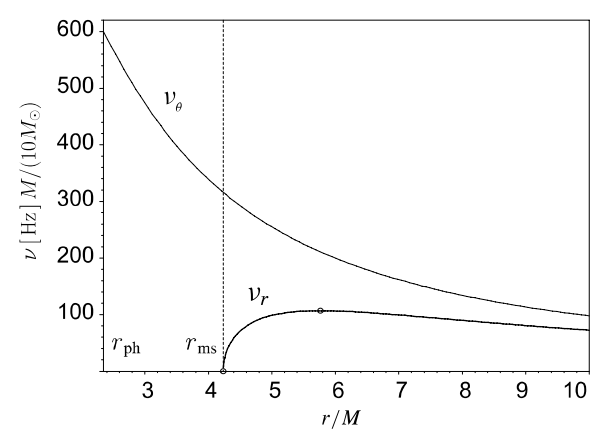

b)

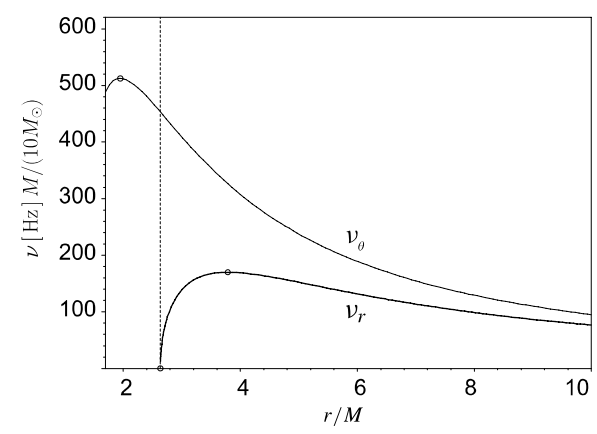

c)

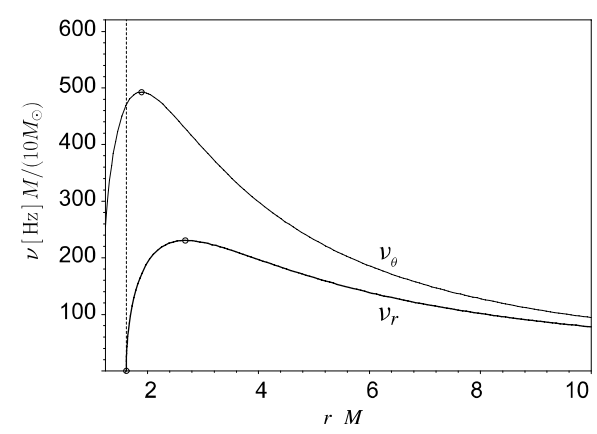

d)

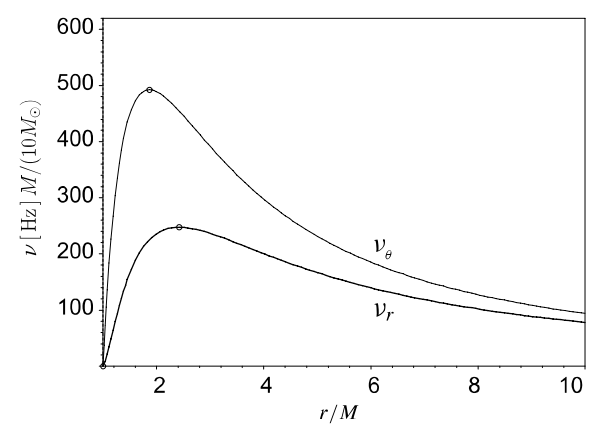

$a=0.5 \in(0,0.748) \equiv \mathrm{BH} 1$

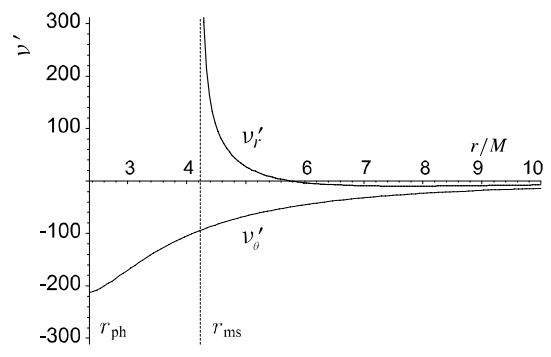

$a=0.85 \in(0.748,0.952) \equiv \mathrm{BH} 2$

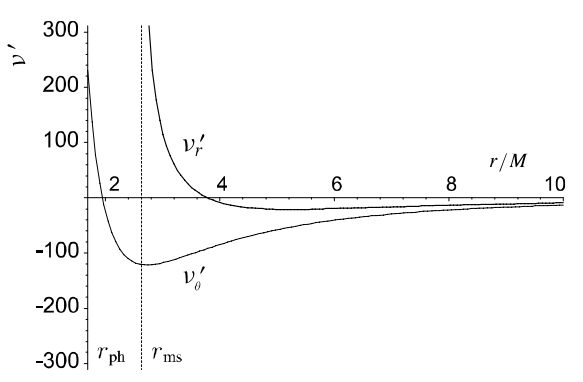

$a=0.98 \in(0.952,1) \equiv \mathrm{BH} 3$

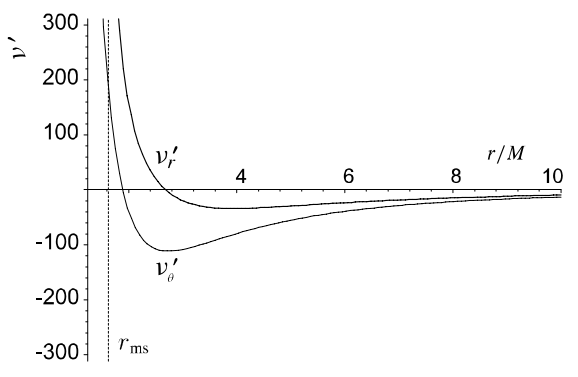

degenerated case of $a=1$

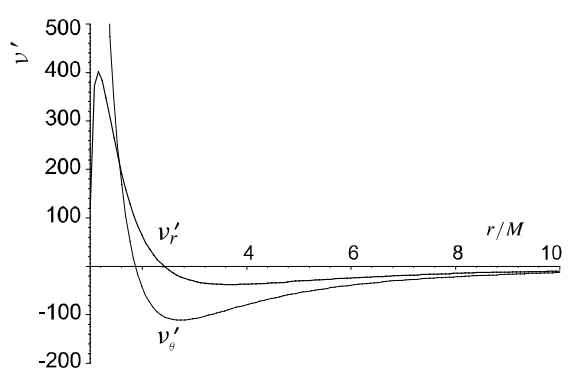

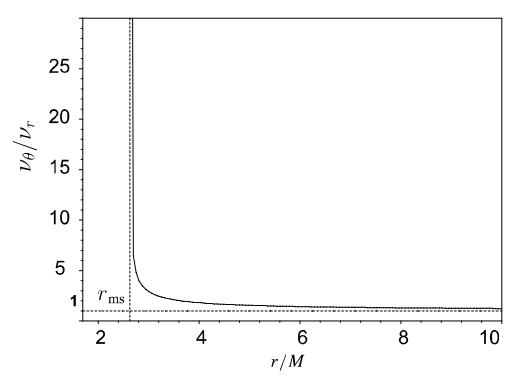
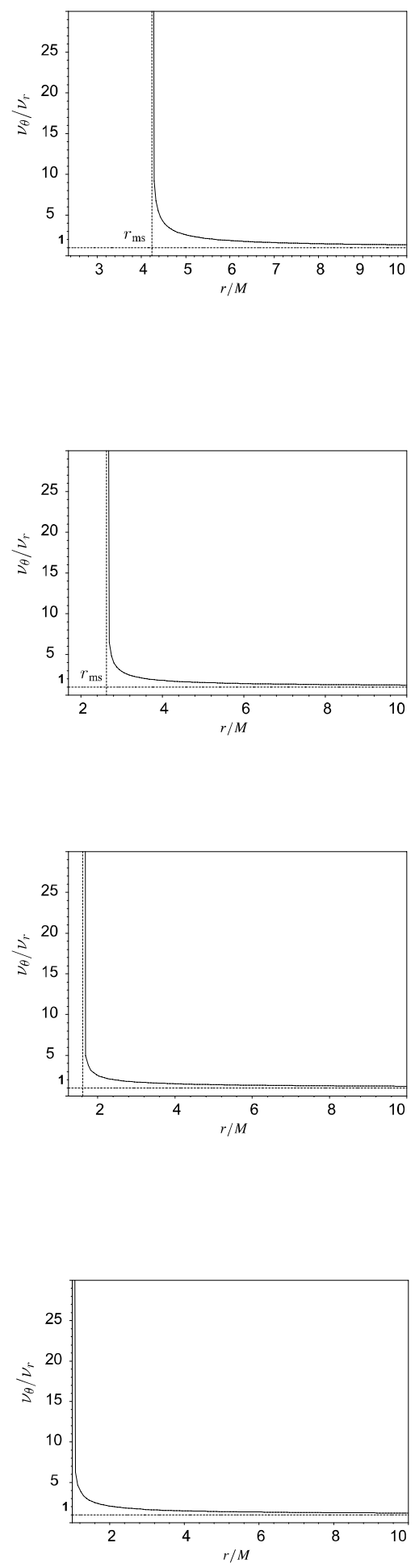

Fig. A.1. Classification of the Kerr black-hole spacetimes. The behaviour of the epicyclic frequencies (left panel), their first derivatives (middle panel), and their ratio $v_{\theta} / v_{\mathrm{r}}$ (right panel) are shown for four representative values of rotational parameter $a$, including the extreme Kerr black hole, the left margin of plots is always situated at the photon circular orbit $r_{\mathrm{ph}}$, while the marginally stable orbit $r_{\mathrm{ms}}$ is denoted by a dashed vertical line. 
a)

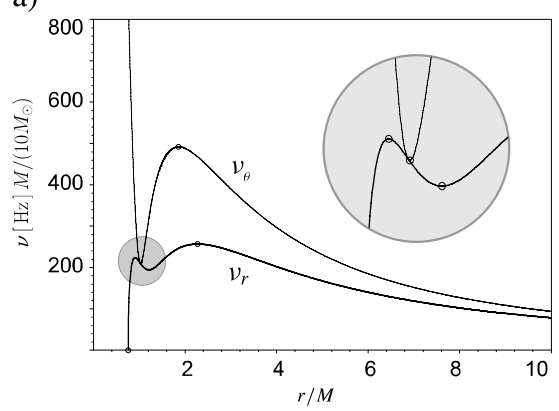

b)

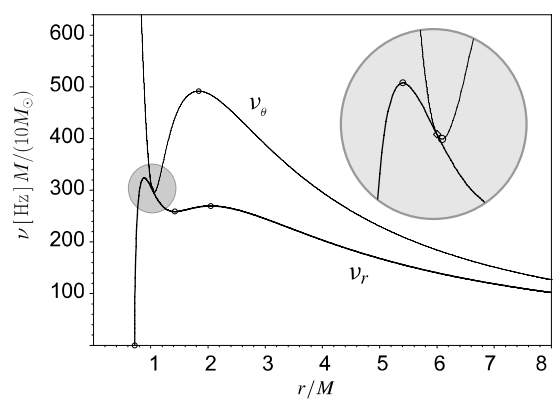

c)

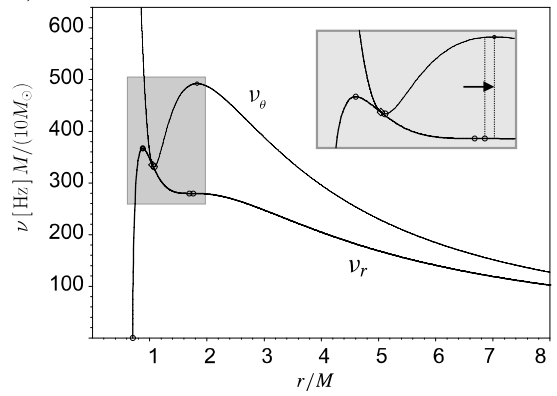

d)

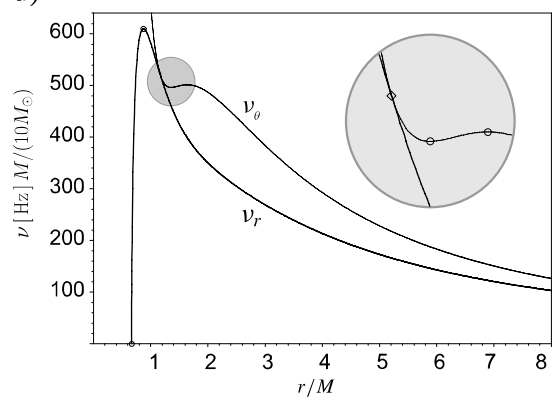

e)

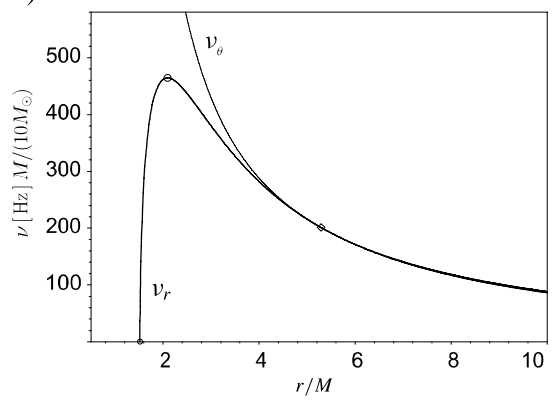

$a=1.009 \in(1,1.012) \equiv \mathrm{NaS} 1$

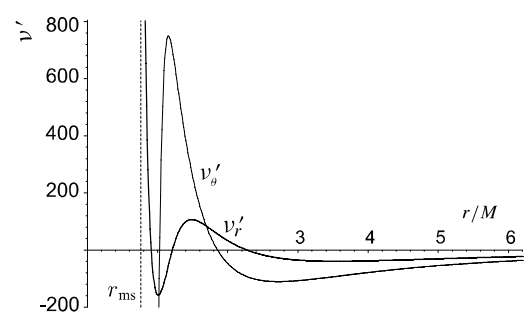

$a=1.019 \in(1.012,1.024) \equiv \mathrm{NaS} 2$

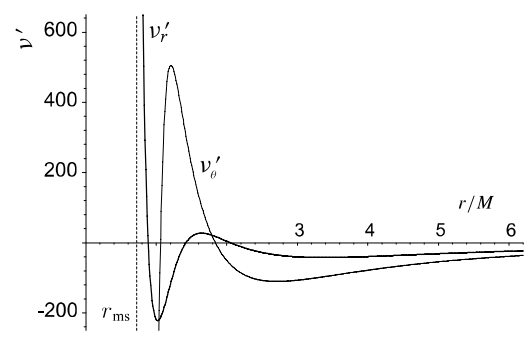

$a=1.0246 \in(1.024,1.025) \equiv \mathrm{NaS} 3$

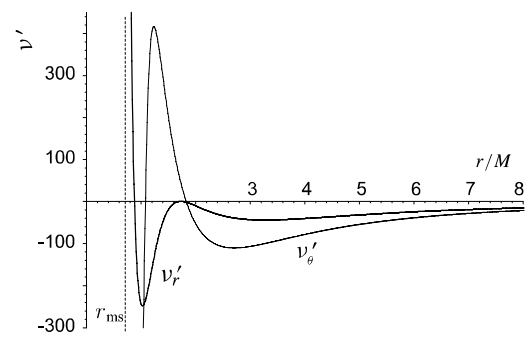

$a=1.08 \in(1.047,1.089) \equiv \mathrm{NaS} 5$

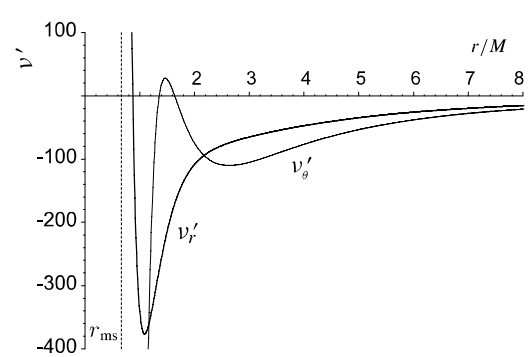

$a=2.3 \in(a>1.089) \equiv \mathrm{NaS} 6$

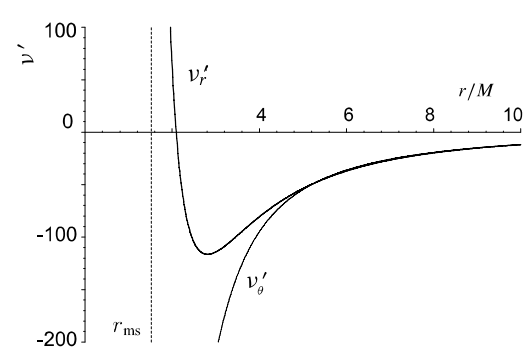

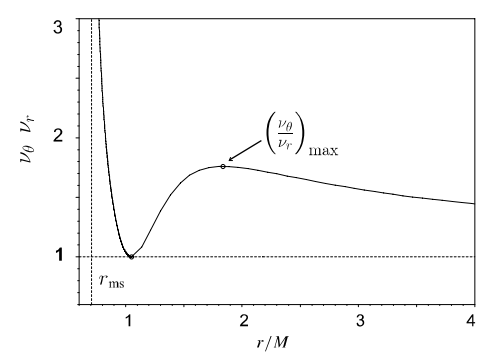
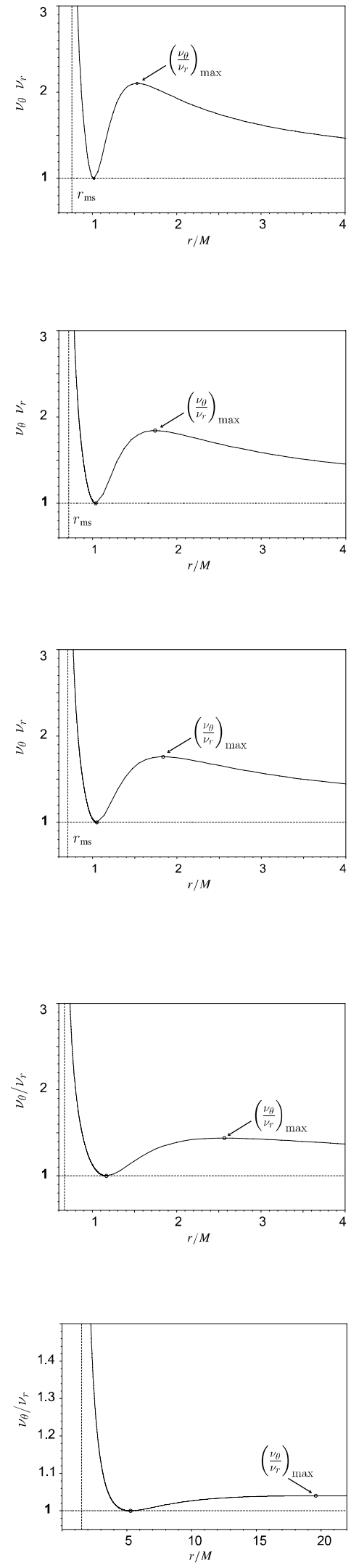

Fig. A.2. Classification of the Kerr naked-singularity spacetimes. The behaviour of the epicyclic frequencies (left panel), their first derivatives (middle panel), and their ratio $v_{\theta} / v_{\mathrm{r}}$ (right panel) are shown for five representative values of rotational parameter $a$. An example of the class NaS4 which differs from the class NaS3 by the absence of the "radial pair" maximum-minimum is not shown. 


\section{References}

Abramowicz, M. A., Bulik, T., Bursa, M., \& Kluźniak, W. 2003, A\&A, 404, L21

Abramowicz, M. A., \& Kluźniak, W. 2001, A\&A, 374, L19

Abramowicz, M. A., \& Kluźniak, W. 2004, in X-ray Timing 2003: Rossi and Beyond, held 3-5 November, 2003 in Cambridge, MA, ed. P. Kaaret, F. K. Lamb, \& J. H. Swank (Melville, NY: American Institute of Physics), AIP Conf. Proc., 714, 21

Abramowicz, M. A., Karas, V., Kluźniak, W., Lee, W., \& Rebusco, P. 2003, Publ. Astr. Soc. Japan, 55, 46

Abramowicz, M. A., Kluźniak, W., Stuchlík, Z., Török, G. 2004, in Proceedings of RAGtime 5: Workshops on black holes and neutron stars, ed. S. Hledík, \& Z. Stuchlík, Opava, 1416/1315 October 2002/03, Silesian University at Opava

Aschenbach, B. 2004, A\&A, accepted [arXiv: astro-ph/0406545]

Bardeen, J. M., Press, W. H., \& Teukolsky, S. A. 1972, ApJ, 178, 347

Bardeen, J. M., 1973 in Black Holes, New York, London, Paris, ed. C. De Witt, \& B. S. De Witt (Gordon and Breach), 215

Belloni, T., Mendez, M., \& Homan, J. 2004, to appear in Proc. NATO/ASI, The Elecromagnetic Spectrum of neutron stars, June 7-18 2004, Marmaris, Turkey, ed. A. Baykal, S. K. Yerli, M. Gilfanov, \& S. Grebenev [arXiv:astro-ph/0409432]

Bursa, M., Abramowicz, M. A., Karas, V., \& Kluźniak, W. 2004, ApJ, 617,45

Carter, B. 1973, in Black Holes, New York, London, Paris, ed. C. De Witt, \& B. S. De Witt (Gordon and Breach), 57

Charlton, N., \& Clarke, C. J. S. 1990, Class. Quantum Grav., 7, 743

Cohen, J. M., \& Gautreau, R. 1979, Phys. Rev. D, 19, 2273

de Felice, F., \& Yu, Y. 1986, Proc. IV Marcell Grossman Meeting ed. R. Ruffini

de Felice, F., \& Yu, Y. 2001, Class. Quantum Grav., 18, 1235

de Villiers, J. P., Hawley, J. F., \& Krolik, J. H. 2003 [arXiv: astro-ph/0307260]

Ford, E., Kaaret, P., Tavani, M., et al. 1997, ApJ, 475, 123

Horák, J., Abramowicz, M. A., Karas, V., \& Kluźniak, W. 2004, PASJ, 56,819

Hubeny, V. E. 1999, Phys. Rev. D, 19, 2273

Igumenshchev, I.V., Narayan, R., \& Abramowicz, M. A. 2003, ApJ, 592,104

Israel, W. 1986 Proc. 14th Yamada Conf., ed. H., Sato, \& T., Nakamura

Joshi, P. S., Dadhich, N., \& Maartens, R. 2002, Phys. Rev. D, 65, 10, 101501

Joshi, P. S., Goswami, R., \& Dadhich, N. 2004, Phys. Rev. D, 70, 8, 087502

Kato, S. 1989, PASJ, 41, 745

Kato, S. 2004a, Publ. Astron. Soc. Japan, 56, 905

Kato, S. 2004b, Publ. Astron. Soc. Japan, 56, 25K

Kato, Y. 2004c, PASJ, 56, 931K

Kato, S., \& Fukue, J. 1980, Publ. Astron. Soc. Japan, 32, 377
Kato, S., Fukue, J., \& Mineshige, S. 1998 (Kyoto University Press)

Kluźniak, W., \& Abramowicz, M. A. 2000, Phys. Rev. Lett., submitted [arXiv:astro-ph/0105057]

Kluźniak, W., Abramowicz, M. A. 2001, Acta Phys. Polonica B, 32, 3605 [http://th-www.if.uj.edu.pl/acta/vol32/t11.htm]

Kluźniak W., Abramowicz, M. A. 2002 [arXiv: astro-ph/0203314]

Kozłowski, M., Jaroszyński, M., \& Abramowicz, M. A. 1978, A\&A, 63, 209

Landau, L. D., \& Lifshitz, E. M. 1976, Mechanics, 3rd edition (Oxford: Pergamon Press)

Krolik, J. H., \& Hawley, J. F. 2002, ApJ, 573, 754

Lake, K., \& Zannias, T. 1990, Phys. Rev. D, 41, 3866

Matsumoto, R., Kato, S., \& Honma, F. 1989, Theory of Accretion Disks, NATO ASIC Proc., 290, 167

McClintock, J. E, \& Remillard, R. A. 2003 [arXiv: astro-ph/0306213]

Miller, J. C., \& de Felice, F. 1985, ApJ, 298, 474

Nakamura, T., Oohara, K., \& Kojima, Y. 1987, Progr. Theoret. Phys. Suppl., 90, 1

Nayfeh, A. H., \& Mook, D. T. 1979, Nonlinear Oscillations (New York: Wiley-Interscience)

Nowak, M., \& Lehr, D. 1999, in Theory of Black Hole Accretion Disks, ed. M. A. Abramowicz, G. Björnsson, \& J. E. Pringle (Cambridge: Cambridge University Press)

Nowak, M. A., \& Wagoner, R. V. 1991, ApJ, 378, 656

Nowak, M. A., \& Wagoner, R. V. 1992, ApJ, 393, 697

Okazaki, A. T., Kato, S., \& Fukue, J. 1987, Publ. Astron. Soc. Japan, 39,457

Penrose, R. 1969, Riv. Nuovo Cimento 1, 252

Perez, C. A., Silbertgleit, A. S., Wagoner, R. V., \& Lehr, D. E. 1997, ApJ, 476, 589

Quinn, T. C., \& Wald, R. M. 1979, Phys. Rev. D., 60, 064009

Rebusco, P. 2004, Laurea Thesis, University of Trieste

Rezzolla, L. 2004, in X-ray Timing 2003: Rossi and Beyond. held $3-$ 5 November, 2003 in Cambridge, MA, ed. P. Kaaret, F. K. Lamb, \& J. H. Swank (Melville NY: American Institute of Physics), AIP Conf. Proc., 714, 36

Stalker, J. G., Shadi Tahvildar-Zadeh, A. 2004, Class. Quantum Grav., 21,2831

Strohmayer, T. E., Zhang, W., Swank, J. H., et al. 1996, ApJ, 468, L9 Stuchlík., Z. 1980, Bull. Astronom. Inst. Czechoslovakia, 31, 129

Thorne, K. S. 1974, ApJ, 191, 507

Török, G., Abramowicz, M. A., Kluźniak, W., \& Stuchlík, Z. 2005, A\&A, accepted [arXiv: astro-ph/0401464]

van der Klis, M. 2000, ARA\&A, 38, 71

van der Klis, M., Wijnands, R. A. D., Horne, K., \& Chen, W. 1997, ApJ, 481, 97

Wald, R. M. 1974, Ann. Phys., 83, 548

Zhang, W., Smale, A. P., Strohmayer, T. E., \& Swank, J. H. 1998, ApJ, 500,171 